\title{
Dynamics of counterpropagating waves in parametrically forced, large aspect ratio, nearly conservative systems with nonzero detuning
}

\author{
José M. Vega , Edgar Knobloch \\ E.T.S.I. Aeronánicos, Lnirersidad Politécnica de Madrid, Plaza Cardenal Cinneros 3, 28040) Madrid. Spain \\ Deparment of Plnsics. Unitersity of Callifmia. Berkeles. CA 94720. CSA \\ Department of Applied Mathematis, Lnitersity of Leeds, Leeds LS2 9JT, UK
}

\begin{abstract}
The dynamics of parametrically driven, slowly varying counterpropagating wave trains in nearly conservative systems are considered. The system is assumed to be invariant under reflection and translations in one direction, and periodic boundary conditions with period $L$ are imposed, with $L$ large but not too large in order that the effect of detuning be significant. The dynamics near the minima of the resulting resonance tongues are described by a system of coupled nonlocal Schrödinger equations with damping and parametric forcing. Elsewhere the long time behavior of the system is described by a damped complex Duffing equation with real coefficients, whose solutions relax to spatially uniform standing waves. Near the bicritical points where two adjacent resonance tongues intersect a pair of coupled damped complex Duffing equations captures the properties of both pure and mixed modes, and of the periodic solutions resulting from a Hopf bifurcation on the branch of mixed modes. As an application, we consider a Faraday system in an annulus in which a pair of counterpropagating surface gravity-capillary waves are excited parametrically by vertical vibration of the container, including the mean flow driven by time-averaged Reynolds stresses due to oscillatory viscous boundary layers along the bottom and the free surface. This mean flow is shown to have a large effect near the bicritical point, where the mean flow changes the dynamics of the system both quantitatively and qualitatively. In particular, inclusion of the mean flow permits Hopf bifurcations from the branches of pure standing waves, and parity-breaking bifurcations from mixed modes.
\end{abstract}

PACS: $47.20 . \mathrm{Ky} ; 47.20 . \mathrm{Ma} ; 47.35 .+\mathrm{i} ; 47.54 .+\mathrm{r}$

Keyrords: Parametric resonance: Gravity-capillary waves; Streaming flow: Asymptotics 


\section{Introduction}

In this paper we consider a pair of coupled, weakly damped, parametrically forced Schrödinger equations that describe the weakly nonlinear evolution of a pair of slowly modulated, counterpropagating wavetrains in extended, nearly conservative systems invariant under reflection and translation in one spatial dimension. Elsewhere (Martel et al., 2000; Higuera et al., 2002b) we have discussed the limit of small detuning, and shown that in this regime the dynamies near onset is described by a pair of coupled nonlocal Schrödinger equations with damping and parametric forcing. These equations describe the dynamics near the minimum of each resonance tongue. However, in systems that are large but not too large the allowed wavenumbers may differ from the wavenumber selected via the dispersion relation and the difference between the response frequency and (half the) forcing frequency may be substantial. In the following we call this difference the detuning and discuss the dynamics when the detuning is nonzero, i.e., the dynamics near the boundaries of the resonance tongues away from their minima.

The present study is motivated by the dynamics of surface gravity-capillary waves excited by the vertical vibration of a container of liquid (Miles and Henderson, 1990; Cross and Hohenberg, 1993). In low viscosity liquids the surface waves are described by a pair of coupled, weakly damped, parametrically forced Schrödinger equations, but these include in addition a coupling to a mean flow driven by time-averaged Reynolds stresses in the oscillatory viscous boundary layers along the bottom (and lateral) boundaries as well as the free surface (Vega et al., 200I). This mean flow was in fact observed already by Faraday (1831) in his classic experiments on this system (since named after him) through the nonuniform accumulation of sand at the bottom of the container. The same effect has also been observed in dust along the wall of a sound tube (the Kundt tube) (Rayleigh, 1945; Schlichting. 1968). A theoretical explanation of this effect was provided by Rayleigh (1883), who showed that the mean flow responsible for this accumulation was viscous in origin (even though the viscosity was quite small). He did so by analyzing the flow in what we nowadays call the oscillatory boundary layer near the bottom of the container, and obtained the key ingredients for subsequent work on this flow by Schlichting (1932). Schlichting's analysis was in turn extended to the viscous boundary layer along the free surface by Longuet-Higgins (1953) (see Craik (1985) for the early references and Riley (2001) for a recent review). This mean flow (also known as a streaming flow, or acoustic streaming) dillers fundamentally from the imiscid mean flow that appears in water wave models such as the celebrated Davey-Stewartson (or Benney-Roskes) equations (Davey and Stewartson, 1974; Pierce and Knobloch, 1994). The viscous mean flow of interest here is a consequence of the nonzero tangential velocity and shear stress at the internal edge of the oscillatory boundary layers attached to the walls and the free surface, respectively, both of which vanish in the inviscid regime. Although mean flows of this type have sometimes been studied as a by-product of surface waves (Liu and Davis, 1977; Iskandarani and Liu, 1991), their dynamical interaction with the surface waves that produce them has been systematically ignored, based on the mistaken assumption that their effects should be of higher order. Recent work on nearly inviscid Faraday waves in a variety of configurations (Vega et al., 2001; Higuera ct al., 2001, 2002a,c; Martín et al., 2002) (sec also Knobloch and Vega (2002), and Knobloch ct al. (2002) for recent reviews) confirms that the mean flow interacts with the surface waves already at leading order, and hence cannot be omitted from the analysis. In particular the mean flow is unavoidable near mode interaction points (cf. Higuera et al., 2002c), the case of particular interest in the present 


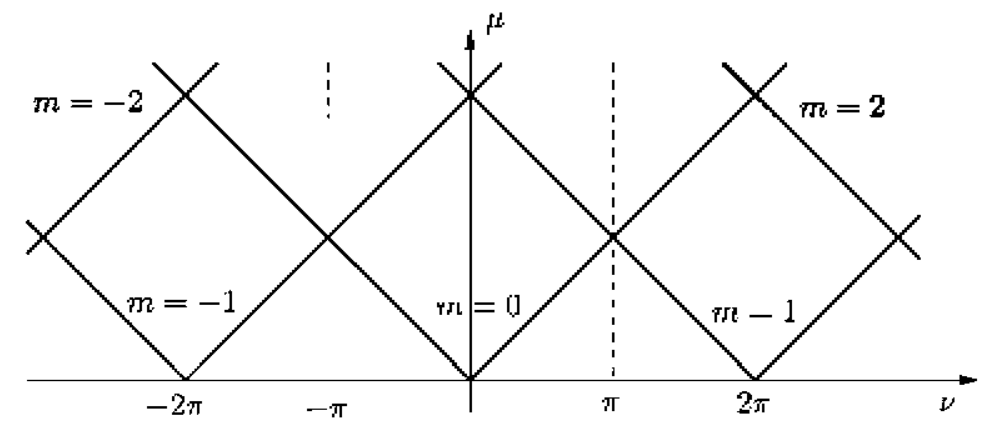

Fig. 1. Resonance tongues $(\mu- \pm(\nu-2 \pi m))$ showing the instability threshold for the flat state. Owing to the invariance of the amplitude equations under the operation $A^{1} \rightarrow i A:, \mu \rightarrow-\mu$ the diagram for $\mu<0$ is obtained via reflection in $\mu-0$.

work. Regrettably, there are at present no experimental studies of the excitation of mean flows near such points in large domains (cf. Douady et al,, 1989, Fig, 1), although mode interactions in small aspect ratio systems have been studied (Ciliberto and Gollub, 1985; Simonelli and Gollub, 1989).

Against this background, the remainder of the paper is organized as follows. The basic amplitude equations governing the evolution of the complex amplitudes of the two counterpropagating waves are summarized in Section 2, together with a bricf summary of the structure of the resonance tongues associated with a parametric instability (Fauve, 1995). The weakly nonlinear dynamics near threshold for generic values of detuning is considered in Section 3, while Section 4 focuses on the dynamics near a bicritical point, where neighboring resonance tongues intersect. The Faraday system is considered in Section 5, followed by concluding remarks in Section 6. In cach case, we derive the leading order amplitude (or amplitude-mean flow) equations and discuss their propertics. It should be emphasized that although in the limit considered in this paper the aspect ratio is large it is not so large that spatial modulations of the surface waves enter into the theory. This is because the theory is developed for forcing amplitudes that are appropriately close to threshold. For larger aspect ratios spatial modulations must be taken into account. This may be achicved via mode truncation as in Decent and Craik (1999), or via a systematic asymptotic expansion focusing on the effects of dispersion as in Martel ct al. (2003) and references therein. The coupling between these effects and the viscous mean flow in a nearly inviscid Faraday system is explored in Lapucrta ct al. (2002). These effects do not enter in the regime studied in the present paper.

\section{Amplitude equations and stability of the flat state}

In an extended nearly conservative system the amplitudes of slowly varying counterpropagating wavetrains, driven parametrically by external vibration, satisfy equations of the form (Martel et al. (2000), Eqs. (5)-(7))

$$
\begin{aligned}
& A_{t}^{ \pm} \mp A_{x}^{ \pm}-\mathrm{i} \mu \bar{A}^{\mp}=-(\delta+\mathrm{i} \varphi) A^{ \pm}+\mathrm{i} \chi A_{x x}^{ \pm}+\mathrm{i}\left(\beta\left|A^{ \pm}\right|^{2}+\gamma\left|A^{\mp}\right|^{2}\right) A^{ \pm}+\cdots, \\
& A^{ \pm}(x+L, t)=A^{ \pm}(x, t) .
\end{aligned}
$$


These equations are valid provided that

$$
\left|A_{x x}^{ \pm}\right| \ll\left|A_{x}^{ \pm}\right| \ll\left|A^{ \pm}\right| \ll 1, \quad\left|A_{t}^{ \pm}\right| \ll\left|A^{ \pm}\right| \ll 1,
$$

and the real parameters $\mu$ (forcing amplitude), $v$ (detuning), and $\delta$ (damping rate) are small. The dispersion cocfficient $\alpha$ and the cocfficients $\beta, \gamma$ of the nonlinear terms are also real but of order unity. In writing these equations we have shifted the phase of $A^{ \pm}$by $\pi / 4$ relative to the equations used in Martel et al. (2000).

These equations are invariant under reflection and translations, represented by the operations

$$
A^{+} \leftrightarrow A^{-} \text {and } A^{ \pm} \rightarrow \mathrm{e}^{ \pm \mathrm{i} r} A^{ \pm} \text {for all } c \text {. }
$$

In fact Eqs. (2.1) and (2.2) remain invariant (after a rescaling to restore the unit group velocity) under the transformation

$$
v \rightarrow v+2 \pi / L-\alpha(2 \pi / L)^{2}, \quad A^{ \pm} \rightarrow \mathrm{e}^{ \pm \mathrm{i} 2 \pi x / L} A^{ \pm} .
$$

This transformation allows us to restrict the detuning to a neighborhood of the interval

$$
-\pi / L<v \leqslant \pi / L
$$

The limit considered in Martel et al. (2000),

$$
\delta \sim v \sim \mu \sim L^{-2} \ll 1
$$

corresponds to (a) a moderately large aspect ratio (so detuning is still felt), (b) a forcing frequency that yields an appropriately small detuning, in addition to (c) an appropriately small threshold acceleration to overcome the (assumed) small dissipation. These conditions restrict the validity of the theory to the vicinity of the minimum of the primary resonance tongue. As shown in Martel et al. (2000) in this regime Eqs. (2.1) and (2.2) reduce to a pair of coupled nonlocal Schrödinger equations. Identical analysis holds near the minima of the other resonance tongues, corresponding to the excitation of modes with modulation wavenumber $m$ ) provided $|v-2 m \pi / L| \sim L^{-2}$. However, this reduction no longer applies if condition (b) is relaxed. It is this case, viz.

$$
|v| \sim L^{-1}, \quad y \neq 0
$$

that is of interest here. In the following we therefore focus on the limit:

$$
\delta L \ll 1, \quad|v| \sim L^{-1} \ll 1, \quad|\mu-v| \ll|v|,
$$

where we are anticipating that the instability threshold is $\mu_{c} \simeq v$. This limit is the counterpart of (2.7) (after relaxing the condition $|v| \ll L^{-1}$ ), except for the fact that we are not making at this stage any assumption about the order of magnitude of the damping rate $\delta$ exeept that it is small compared to $L^{-1}$.

To study the limit (2.9) we scale variables and parameters according to

$$
(x, l)=L(\tilde{x}, \tilde{l}), \quad A^{ \pm}=L^{-1} \tilde{A}^{ \pm}, \quad(v, \mu)=L^{-1}(\tilde{v}, \tilde{u}) .
$$

Dropping the tildes we obtain (cf. Martel et al., 2000, Eqs. (11) and (12))

$$
\begin{aligned}
& A_{l}^{ \pm} \mp A_{x}^{ \pm}+\mathrm{i}\left(\nu A^{ \pm}-\mu \bar{A}^{\mp}\right)=-\partial L A^{ \pm}+\mathrm{i} L^{-1}\left[\left(\beta\left|A^{ \pm}\right|^{2}+\gamma\left|A^{\mp}\right|^{2}\right) A^{ \pm}+\not A_{x x}^{ \pm}\right]+\cdots, \\
& A^{ \pm}(x+1, t) \equiv A^{ \pm}(x, t) .
\end{aligned}
$$


It is these equations that are the subject of Section 3. We emphasize that the properties of these equations differ from those obtained on the basis of fundamentally small aspect ratio theorics, even for the primary standing waves. This is a consequence of the scaling $(2.10 \mathrm{~b})$ which limits the theory that follows to smaller amplitudes than those accessible in small aspect ratio theories. In particular, we do not expect to see secondary saddle-node bifurcations on the standing wave branches, although our results do reproduce the direction of branching of the primary standing waves from such theories (cf. Umeki, 1991). Additional differences, arising from the presence of a large number of modes in the present system, are discussed in Section 3.

The linear stability of the flat solution $A^{ \pm}=0$ of (2.11) and (2.12) is analyzed by considering infinitesimal perturbations of the form $\exp (\lambda t)$ with a wavenumber $2 \pi m$. The dispersion relation is

$$
(\lambda+\delta L)^{2}+\left[v-2 \pi m+\alpha(2 \pi m)^{2} L^{-1}\right]^{2}-\mu^{2}=0 .
$$

Thus the threshold for the instability of the flat state is associated with the mode $m=0$ (see Fig, 1), and is given by $\mu=v+\ddot{\partial}^{2} L^{2} /(2 v)+\cdots$ (i.e., according to (2.9a) and the rescaling (2.10), $\mu \approx v$, as anticipated above). The remaining (infinitely many) modes are weakly damped (because $\delta L \ll 1$ ) and thus cannot be ignored a priori. These are either of the form

$$
A^{ \pm}=A_{m}^{ \pm} \mathrm{e}^{ \pm \mathrm{i}\left(y_{m} t \mid 2 \pi m x\right)} \quad \text { with } A_{m}^{-}=A_{m}^{\prime} \bar{A}_{m}^{\prime}
$$

or of the form

$$
A^{ \pm}=A_{m}^{ \pm} \mathrm{e}^{ \pm \mathrm{i}\left(-s_{m} t+2 \pi m x\right)} \quad \text { with } A_{m}^{-}=A_{m}^{-} \bar{A}_{m}^{-} \equiv \bar{A}_{m}^{+} / \Lambda_{m}^{-}
$$

where to leading order (as $|\mu-v| \ll|v|, L \rightarrow \infty$, and $\delta L \rightarrow 0$ )

$$
s_{m}=\left[(2 \pi m)^{2}-4 \pi m v\right]^{1 / 2} \quad \text { and } \quad A_{m}^{ \pm}=\frac{ \pm s_{m}-2 \pi m+v}{v} \equiv \frac{[1-v /(m \pi)]^{1 / 2} \mp 1}{[1-v /(m \pi)]^{1 / 2} \pm 1},
$$

for each integer $m \neq 0$. In (2.15) we are using the identity

$$
\Lambda_{m}^{+} \Lambda_{m}^{-}=1 \text {, }
$$

which follows from (2.16). Note that

$$
\left|\Lambda_{m}^{\mid}\right|<1 \quad \text { for all } m \neq 0 \quad \text { if } \quad v \neq 0, \quad \text { and } \quad A_{m} \rightarrow 0 \quad \text { as } m \rightarrow \infty
$$

Thus in the generic case $(v \neq 0, \pi)$ a single marginally unstable mode $(m=0)$ is present at threshold. However, in the special case $v=\pi$ the marginal stability curves for the modes $m=0, m=1$ cross at $\mu=\pi$. In the following we call the point $(v, \mu)=(\pi, \pi)$ a bicritical point or a codimension-two point (see Fig. 1). In the vicinity of this point two modes are near marginal stability and hence nonlinear interactions between them may occur already at small amplitude. Similarly, when $v=0$ the marginal stability curves for $m= \pm 1$ cross at $\mu=2 \pi$. Thus the point $(\nu, \mu)=(0,2 \pi)$ defines another bicritical point, although this point is of less interest since all the local dynamics it produces are necessarily unstable due to the prior instability of the $m=0$ mode. The results for other bicritical points follow from invariance of the amplitude equations under $(2.5)$. Thus near $(y, \mu)$ of the form $(\pi+2 m \pi, \pi)$ two adjacent nearly marginal modes cocxist with an infinite number of weakly damped modes but no other unstable modes. The role played by these weakly damped modes in this region depends on the assumed damping rate, as discussed next. 


\section{Dynamics in the generic case: $v \neq 0, \pi$}

In order to understand the nature of the limit (2.9) and the origin of the complexity that must be expected, we consider two eases, depending on the order of magnitude of the damping rate.

\subsection{Significant damping: $\delta \sim L^{-3 / 2}$}

For this case we introduce the damping rate parameter $\hat{j}$, the bifurcation parameter $\hat{\mu}$, and a slow time variable $\tau$ defined by

$$
\delta=L^{-3 / 2} \hat{\delta}, \quad \mu=v+L^{-1} \hat{\mu}, \quad \text { and } \tau=L^{-1 / 2} l,
$$

and seek a solution in terms of the eigenmodes (2.14) and (2.15):

$$
\begin{aligned}
A^{ \pm}= & A_{00}^{ \pm}+L^{-1 / 2} A_{01}^{ \pm}+L^{-1} A_{02}^{ \pm}+\cdots \\
& +\sum_{0 / m \sim 1} \mathrm{e}^{ \pm \mathrm{i} 2 \pi m x}\left[\left(A_{m}^{ \pm}+L^{-1 / 2} A_{m 1}^{ \pm}+\cdots\right) \mathrm{e}^{1 \beta_{m} t}+\left(B_{m}^{ \pm}+L^{-1 / 2} B_{m 1}^{ \pm}+\cdots\right) \mathrm{e}^{-\mathrm{i} \xi_{m} t}\right] .
\end{aligned}
$$

Here, according to (2.14) and (2.15), the complex amplitudes $A_{00}^{ \pm}, A_{m}^{ \pm}$and $B_{m}^{ \pm}$are such that

$$
A_{000}^{-}=\bar{A}_{00}^{+}, \quad B_{m}^{ \pm}=A_{m}^{+} \bar{A}_{m}^{\mp} .
$$

These and the higher order terms $A_{m j}^{ \pm}$and $B_{m j}^{ \pm}$depend only on the slow time variable $\tau$. Substituting (3.1) and (3.2) into (2.11) and setting to zero the coefficients of $\mathrm{e}^{ \pm \mathrm{i} s_{m} t \pm \mathrm{i} 2 \pi m x}$ at order $O\left(L^{-1 / 2}\right)$ we obtain

$$
\begin{aligned}
& \operatorname{iv}\left(A_{0 \mathrm{l}}^{ \pm}-\bar{A}_{01}^{\mp}\right)=-\mathrm{d} A_{00}^{ \pm} / \mathrm{d} \tau-\hat{\delta} A_{00}^{ \pm}, \\
& \operatorname{iv}\left(A_{m}^{+} A_{m 1}^{ \pm}-\bar{B}_{m 1}^{\mp}\right)=-\mathrm{d} A_{m}^{ \pm} / \mathrm{d} \tau-\hat{\delta} A_{m}^{ \pm}, \\
& \operatorname{iv}\left(A_{m}^{-} B_{m 1}^{ \pm}-\bar{A}_{m 1}^{\mp}\right)=-\mathrm{d} B_{m}^{ \pm} / \mathrm{d} \tau-\hat{\delta} B_{m}^{ \pm} .
\end{aligned}
$$

Eq. (3.3a) now shows that the two equations (3.4) are identical. Thus (3.4) yiclds no solvability condition. However, Eqs. (3.5) and (3.6) do require a solvability condition. Using (2.17) this condition can be written in the form

$$
\left[1-\left(A_{m}^{+}\right)^{2}\right]\left(\mathrm{d} A_{m}^{ \pm} / \mathrm{d} \tau+\hat{\partial} A_{m}^{ \pm}\right)=0
$$

implying that

$$
A_{m}^{ \pm} \rightarrow 0 \text { exponentially as } \tau \rightarrow \infty \text {. }
$$

This property of the solution is a consequence of the magnitude of the damping rate selected by (3.1), cf. (2.9). Thus, after a transient, we can take

$$
A_{m}^{ \pm}=0 \text { for all } m \neq 0 \text {. }
$$

This fact, together with (3.3a), results at order $O\left(L^{-1}\right)$ in the pair of equations

$$
\mathrm{i} \nu\left(A_{02}^{ \pm}-\bar{A}_{02}^{\mp}\right)=-\mathrm{d} A_{01}^{ \pm} / \mathrm{d} \tau-\hat{\delta} A_{01}^{ \pm}+\mathrm{i} \hat{\mu} \bar{A}_{00}^{\mp}+\mathrm{i}(\beta+\gamma)\left|A_{00}^{ \pm}\right|^{2} A_{00}^{ \pm} .
$$


The resulting solvability condition can be written in the form

$$
\mathrm{d}\left(A_{01}^{+}-\bar{A}_{01}^{-}\right) / \mathrm{d} \tau+\hat{\hat{\gamma}}\left(A_{01}^{+}-\bar{A}_{01}^{-}\right)=2 \mathrm{i}\left[\hat{\mu}+(\beta+\gamma)\left|A_{000}^{-}\right|^{2}\right] A_{00}^{+},
$$

or, invoking (3.4) and denoting $A_{00}^{+}=A$,

$$
A^{\prime \prime}+2 \hat{\partial} A^{\prime}+\hat{\delta}^{2} A=2 v\left[\hat{\mu}+(\beta+\gamma)|A|^{2}\right] A \text {. }
$$

In the following we exclude the codimension-two point $\beta+\gamma=0$ and consider therefore the two cases $v(\beta+\gamma)>0$, and $v(\beta+\gamma)<0$. In the former Eq. (3.12) possesses solutions that blow up in finite time, an observation consistent with the fact that the primary bifurcation from the flat state to the simplest standing waves (steady states of (3.12)) is subcritical. In contrast if

$$
v(\beta+\gamma)<0
$$

as assumed henceforth, then all solutions of (3.12) are uniformly bounded as $\tau \rightarrow \infty$. This result follows from the exact relation:

$$
\frac{\mathrm{d}}{\mathrm{d} \tau}\left[\left|A^{\prime}\right|^{2}+\left(\hat{\delta}^{2}-2 v \hat{\mu}\right)|A|^{2}-v(\beta+\gamma)|A|^{4}\right]=-4 \hat{\delta}\left|A^{\prime}\right|^{2},
$$

obtained on multiplying (3.12) by $\bar{A}^{\prime}$ and adding the result to its complex conjugate.

For convenience we write Eq. (3.12) in terms of the amplitude and phase of $A$, defined by $A=R \mathrm{e}^{\mathrm{i} \theta}$,

$$
\begin{aligned}
& R^{\prime \prime}+2 \hat{\delta} R^{\prime}+\left(\hat{\delta}^{2}-\theta^{\prime 2}\right) R=2 v\left[\hat{\mu}+(\beta+\gamma) R^{2}\right] R, \\
& \theta^{\prime \prime}+2\left(\hat{\delta}+R^{\prime} / R\right) \theta^{\prime}=0 .
\end{aligned}
$$

Integrating (3.16) we obtain

$$
\theta^{\prime}=K_{0} R^{-2} \mathrm{e}^{-2 \hat{\hat{\delta}_{\tau}}}
$$

(for some constant $K_{0}$ ), which implies that $\theta^{\prime} \rightarrow 0$ exponentially, as $\tau \rightarrow \infty$. The apparent breakdown of this argument as $R \rightarrow 0$ can be overcome using an equation obtained by summing the product of (3.12) with $\bar{A}$, and its complex conjugate, and integrating the result: $\bar{A} A^{\prime}-A \bar{A}^{\prime}=K_{0} \mathrm{c}^{-2 \hat{\partial} \tau}$, cf. (3.17). Thus for large times we may take the phase of $A$ to be constant and, in particular, take $A$ to be real. Eq. (3.12) then reduces to the standard Duffing equation, whose solutions converge to a steady state at large times. Thus, no complexity can be expected in this case.

Note that if

$$
L^{-1 / 2} \ll \hat{\hat{b}} \ll 1
$$

(the first condition being required for the validity of conditions (3.8)) Eq. (3.12) will evolve in a nondissipative manner over a time of order of $\tau \sim \hat{\delta}^{-1}$ as described by the conservative complex Duffing equation

$$
A^{\prime \prime}-2 v\left[\hat{\mu}+(\beta+\gamma)|A|^{2}\right] A=0 .
$$

The counterpart of (3.14) now yields

$$
\left|A^{\prime}\right|^{2}-2 v \hat{\mu}|A|^{2}-v(\beta+\gamma)|A|^{4}=\text { constant. }
$$

A second integral is given by the counterpart of (3.17), namely

$$
\theta^{\prime}=K_{0} R^{-2},
$$


for some constant $K_{0}$, gencrically nonzero. Thus

$$
R^{\prime \prime}-K_{0}^{2} R^{-3}=2 v\left[\hat{\mu}+(\beta+\gamma) R^{2}\right] R,
$$

and hence only steady states and periodic solutions are to be expected at large times. In fact, Eq. (3.22) can be integrated once, yielding

$$
R^{\prime 2}-K_{1}^{2}=-K_{0}^{2} R^{-2}+\nu\left[2 \hat{\mu}+(\beta+\gamma) R^{2} / 2\right] R^{2},
$$

for some constant $K_{1}$. The allowed time-periodic solutions are now easily computed, but all ultimately decay to steady states for $\tau \gg \hat{\delta}^{-1}$.

\subsection{Sinall damping: $\delta \sim L^{-2}$}

The simplification described in the preceding section is a consequence of the assumption that $\hat{\hat{\delta}} \neq 0$ (in fact, $\hat{\delta} \geqslant L^{-1 / 2}$, or equivalently that $\delta \geqslant L^{-2}$ ), for otherwise (3.8) no longer follows from (3.7). The situation is more interesting for smaller damping rates. Consequently, in this section we consider the case $\delta \sim L^{-2}$, which in conjunction with (2.9) is the true counterpart of (2.7). To study this case we retain the parameter $\hat{\mu}$ defined in (3.1) as the bifurcation parancter, but redefine the damping rate parameter $\hat{\delta}$ and the slow time variable $\tau$ :

$$
\delta=L^{-2} \hat{\delta}, \quad \tau=L^{-1} t .
$$

In fact, we should use two slow time variables in this limit, namely $t \sim L^{1 / 2}$ and $t \sim L$, but this makes the analysis unnecessarily involved. Of course, because only the slowest time variable is used, some of the equations (Eq. (3.30) below) will depend on the small parameter $L^{-1}$. The expansions (3.2) must be replaced by

$$
\begin{aligned}
A^{ \pm}= & A_{00}^{ \pm}+L^{-1} A_{01}^{ \pm}+L^{-2} A_{02}^{ \pm}+\cdots \\
& +\sum_{0 \neq m \sim 1} \mathrm{e}^{ \pm \mathrm{i} 2 \pi m x}\left[\left(A_{m}^{ \pm}+L^{-1} A_{m 1}^{ \pm}+\cdots\right) \mathrm{e}^{\mathrm{i}_{s_{m} l}}+\left(B_{m}^{ \pm}+L^{-1} B_{m 1}^{ \pm}+\cdots\right) \mathrm{c}^{-\mathrm{i} s_{m} l}\right],
\end{aligned}
$$

where the relations (3.3) still hold. Eqs. (3.5) and (3.6) now become

$$
\begin{aligned}
\mathrm{i} v\left(A_{m}^{+} A_{m 1}^{ \pm}-\bar{B}_{m 1}^{\mp}\right)= & -\mathrm{d} A_{m}^{ \pm} / \mathrm{d} \tau-\hat{\delta} A_{m}^{ \pm}+\mathrm{i}\left[\hat{\mu} \bar{B}_{m}^{\mp}-(2 \pi m)^{2} \alpha A_{m}^{ \pm}\right]+\mathrm{i}|A|^{2}\left[(2 \beta+\gamma) A_{m}^{ \pm}+\gamma \bar{B}_{m}^{\mp}\right] \\
& -\left.\mathrm{i} \beta A_{m}^{ \pm}\right|^{2} A_{m}^{ \pm}+2 \mathrm{i} \beta \sum_{n / 0}\left(\left|A_{n}^{ \pm}\right|^{2}+\left|B_{n}^{ \pm}\right|^{2}\right) A_{m}^{ \pm} \\
& +\mathrm{i} \gamma \sum_{n \neq 0}\left[\left(\left|A_{n}^{\mp}\right|^{2}+\left|B_{n}^{\mp}\right|^{2}\right) A_{m}^{ \pm}+\left(A_{n}^{\mp} B_{n}^{ \pm}+A_{n}^{ \pm} B_{n}^{\mp}\right) \bar{B}_{m}^{\mp}\right], \\
\mathrm{i} v\left(A_{m}^{-} B_{m 1}^{ \pm}-\bar{A}_{m 1}^{\mp}\right)= & -\mathrm{d} B_{m}^{ \pm} / \mathrm{d} \tau-\hat{\delta} B_{m}^{ \pm}+\mathrm{i}\left[\hat{\mu} \bar{A}_{m}^{\mp}-(2 \pi m)^{2} \not B_{m}^{ \pm}\right]+\mathrm{i}|A|^{2}\left[(2 \beta+\gamma) B_{m}^{ \pm}+\gamma \overline{A_{m}^{\mp}}\right] \\
& -\mathrm{i} \beta\left|B_{m}^{ \pm}\right|^{2} B_{m}^{ \pm}+2 \mathrm{i} \beta \sum_{n / 0}\left(\left|A_{n}^{ \pm}\right|^{2}+\left|B_{n}^{ \pm}\right|^{2}\right) B_{m}^{ \pm} \\
& +\mathrm{i} \gamma \sum_{n \neq 0}\left[\left(\left|A_{n}^{\mp}\right|^{2}+\left|B_{n}^{\mp}\right|^{2}\right) B_{m}^{ \pm}+\left(A_{n}^{\mp} B_{n}^{ \pm}+A_{n}^{ \pm} B_{n}^{\mp}\right) \bar{A}_{m}^{\mp}\right],
\end{aligned}
$$


where $A=A_{00}^{+}\left(=\bar{A}_{00}^{-}\right)$, as above. The solvability conditions for these equations can be written, using (3.3b), in the form

$$
\begin{aligned}
{[1} & \left.-\left(A_{m}^{+}\right)^{2}\right]\left(\mathrm{d} A_{m}^{ \pm} / \mathrm{d} \tau+\hat{\delta} A_{m}^{ \pm}\right)=2 \mathrm{i} \hat{\mu} A_{m}^{+} A_{m}^{ \pm}-\mathrm{i} \alpha\left[1+\left(A_{m}^{+}\right)^{2}\right](2 \pi m)^{2} A_{m}^{ \pm} \\
& +\mathrm{i}\left((2 \beta+\gamma)\left[1+\left(A_{m}^{\perp}\right)^{2}\right]+2 \gamma A_{m}^{!}\right)|A|^{2} A_{m}^{ \pm}-\mathrm{i} \beta\left[1+\left(A_{m}^{\mid}\right)^{4}\right]\left|A_{m}^{ \pm}\right|^{2} A_{m}^{ \pm} \\
& +2 \mathrm{i} \beta \sum_{n \neq 0}\left(\left[1+\left(A_{m}^{+} A_{n}^{+}\right)^{2}\right]\left|A_{n}^{ \pm}\right|^{2}+\left[\left(A_{m}^{+}\right)^{2}+\left(A_{n}^{+}\right)^{2}\right]\left|A_{n}^{\mp}\right|^{2}\right) A_{m}^{ \pm} \\
& +\mathrm{i} \gamma \sum_{n \neq 0}\left[\left(\Lambda_{m}^{+}+\Lambda_{m}^{+}\right)^{2}\left|A_{n}^{ \pm}\right|^{2}+\left(1+A_{m}^{-} A_{m}^{+}\right)^{2}\left|A_{n}^{\mp}\right|^{2}\right] A_{m}^{ \pm}
\end{aligned}
$$

implying that

$$
\left[1-\left(\Lambda_{m}^{+}\right)^{2}\right]\left(\mathrm{d}\left|A_{m}^{ \pm}\right|^{2} / \mathrm{d} \tau+2 \hat{\partial}\left|A_{m}^{ \pm}\right|^{2}\right)=0 .
$$

Thus once again $\left|A_{m}^{ \pm}\right| \rightarrow 0$ for all $m \neq 0$, albeit on the slower timescale $\tau \sim 1$. With this simplification the counterpart of Eq. (3.12) becomes

$$
L^{-1}\left(A^{\prime \prime}+2 \hat{\delta} A^{\prime}+\hat{\delta}^{2} A\right)=2 v\left[\hat{\mu}+(\beta+\gamma)|A|^{2}\right] A,
$$

and the analysis of Section 3.1 carries over verbatim to the present case. In particular no persistent complex dynamics are expected as $t \rightarrow \infty$. Thus contrary to expectation the $\delta \sim L^{-2}$ case is identical to the $\delta \sim L^{-3 / 2}$ case, except for the (considerably longer) timescale for the decay of complex transients.

\section{Dynamics near the bicritical point: $|v-\pi| \ll 1$}

Let us consider now the dynamics near the codimension-two point obtained at $v=\pi$. For simplicity, we consider the limit of significant damping. Smaller values of the damping ratio only lead to complex transients, as in Section 3.2. We introduce the bifurcation parameters $\hat{\mu}$ and $\hat{v}$, defined as

$$
\mu=\pi+L^{-1}\left(\hat{\mu}-2 \pi^{2} \alpha\right), \quad v=\pi+L^{-1}\left(\hat{v}-2 \pi^{2} \alpha\right)
$$

where the shift $-2 \pi^{2} \alpha$ is included to eliminate the effect of dispersion (see below), and use the same scaling and time variables as in Section 3.1, namely

$$
\delta=L^{-3 / 2} \hat{\delta}, \quad \tau=L^{-1 / 2} t .
$$

Eqs. (2.11) and (2.12) now become

$$
\begin{aligned}
& A_{t}^{ \pm} \mp A_{x}^{ \pm}+\mathrm{i} \pi\left(A^{ \pm}-\bar{A}^{\mp}\right)=\left.-L^{-1 / 2}\left(A_{\tau}^{ \pm}+\hat{\delta} A^{ \pm}\right)+\mathrm{i} L^{-1}\left[\left(\hat{\mu}-2 \pi^{2} \alpha\right) \overline{A^{\mp}}-\left(\hat{v}-2 \pi^{2} \alpha\right) A^{ \pm}\right)\right] \\
&+\mathrm{i} L^{-1}\left[\left(\beta\left|A^{ \pm}\right|^{2}+\gamma\left|A^{\mp}\right|^{2}\right) A^{ \pm}+\alpha A_{x x}^{ \pm}\right]+\cdots \\
& A^{ \pm}(x+1, t) \equiv A^{ \pm}(x, t) .
\end{aligned}
$$

The eigenfrequencies and the constants defined in (2.16) are now given by

$$
s_{m}=2 \pi\left(m^{2}-m\right)^{1 / 2}, \quad \Lambda_{m}^{ \pm}=\left( \pm s_{m}-2 m \pi+\pi\right) / \pi,
$$


and the eigenfrequencies therefore coincide in pairs, namely

$$
s_{0}=s_{1}=0, \quad s_{-1}=s_{2}=2 \sqrt{2} \pi, \quad s_{-2}=s_{3}=2 \sqrt{6} \pi, \ldots .
$$

Since the marginal modes correspond to $m=0, m=1$ we may write for (3.2)

$$
A^{ \pm}=A_{0}^{ \pm}+L^{-1 / 2} A_{1}^{ \pm}+L^{-1} A_{2}^{ \pm}+\cdots+\left(B_{0}^{ \pm}+L^{-1 / 2} B_{1}^{ \pm}+L^{-1} B_{2}^{ \pm}+\cdots\right) \mathrm{c}^{ \pm 2 \mathrm{i} \pi x}+H O H
$$

where, as in Section 3.1, we have

$$
A_{0}^{-}=\bar{A}_{0}^{+}, \quad B_{0}^{-}=-\bar{B}_{0}^{+},
$$

and the abbreviation $H O H$ stands for spatial harmonics with wavenumbers $2 \pi m$, with $m \neq 0,1$. As in Section 3 these higher harmonics decay to zcro exponentially as $\tau \rightarrow \infty$ and can be ignored. Substitution of (4.7) into (4.3) yiclds, at orders $O\left(L^{-1 / 2}\right)$ and $O\left(L^{-1}\right)$,

$$
\begin{aligned}
& \mathrm{i} \pi\left(A_{1}^{ \pm}-\bar{A}_{1}^{\mp}\right)=-\mathrm{d} A_{0}^{ \pm} / \mathrm{d} \tau-\hat{\delta} A_{0}^{ \pm}, \\
& \mathrm{i} \pi\left(-B_{1}^{ \pm}-\bar{B}_{1}^{\mp}\right)=-\mathrm{d} B_{0}^{ \pm} / \mathrm{d} \tau-\hat{\delta} B_{0}^{ \pm}
\end{aligned}
$$

and

$$
\begin{aligned}
\mathrm{i} \pi\left(A_{2}^{ \pm}-\bar{A}_{2}^{\mp}\right)= & -\mathrm{d} A_{1}^{ \pm} / \mathrm{d} \tau-\hat{\delta} A_{1}^{ \pm}+\mathrm{i}\left(\hat{\mu}-2 \pi^{2} \alpha\right) \bar{A}_{0}^{\mp}-\mathrm{i}\left(\hat{v}-2 \pi^{2} \alpha\right) A_{0}^{ \pm} \\
& +\mathrm{i} \beta\left(\left|A_{0}^{ \pm}\right|^{2}+2\left|B_{0}^{ \pm}\right|^{2}\right) A_{0}^{ \pm}+\mathrm{i} \gamma\left[\left(\left|A_{0}^{\mp}\right|^{2}+\left|B_{0}^{\mp}\right|^{2}\right) A_{0}^{ \pm}+\bar{A}_{0}^{\mp} B_{0}^{\mp} B_{0}^{ \pm}\right], \\
\mathrm{i} \pi\left(-B_{2}^{ \pm}-\bar{B}_{2}^{\mp}\right)= & -\mathrm{d} B_{1}^{ \pm} / \mathrm{d} \tau-\hat{\delta} B_{1}^{ \pm}+\mathrm{i}\left(\hat{\mu}-2 \pi^{2} \alpha\right) \bar{B}_{0}^{\mp}-\mathrm{i}\left(\hat{v}+2 \pi^{2} \alpha\right) B_{0}^{ \pm} \\
& +\mathrm{i} \beta\left(\left|B_{0}^{ \pm}\right|^{2}+2\left|A_{0}^{ \pm}\right|^{2}\right) B_{0}^{ \pm}+\mathrm{i} \gamma\left[\left(\left|B_{0}^{\mp}\right|^{2}+\left|A_{0}^{\mp}\right|^{2}\right) B_{0}^{ \pm}+\bar{B}_{0}^{\mp} A_{0}^{\mp} A_{0}^{ \pm}\right] .
\end{aligned}
$$

Using (4.8) we obtain that Eqs. (4.9) and (4.10) are always solvable. But, as in Scetion 3.1, Eqs. (4.11) and (4.12) possess a solution only if two solvability conditions hold. These lead to the following evolution equations for $A \equiv A_{0}^{-}=\bar{A}_{0}^{-}$and $B \equiv B_{0}^{+}=-\bar{B}_{0}^{-}$:

$$
\begin{aligned}
& A^{\prime \prime}+2 \hat{\delta} A^{\prime}+\hat{\delta}^{2} A=2 \pi\left[\hat{\mu}-\hat{v}+\beta\left(|A|^{2}+2|B|^{2}\right)+\hat{\gamma}|A|^{2}\right] A, \\
& B^{\prime \prime}+2 \hat{\delta} B^{\prime}+\hat{\delta}^{2} B=2 \pi\left[\hat{\mu}+\hat{v}-\beta\left(|B|^{2}+2|A|^{2}\right)-\gamma|B|^{2}\right] B .
\end{aligned}
$$

Note that these equations are invariant under the operations

$$
A \rightarrow-A, \quad A \rightarrow \mathrm{e}^{\mathrm{i} c_{1}} A \quad \text { for all } c_{1}, \quad B \rightarrow-B, \quad \text { and } \quad B \rightarrow \mathrm{e}^{\mathrm{i} c_{2}} B \text { for all } c_{2},
$$

which generate the group $\mathrm{O}(2) \times \mathrm{O}(2)$, much larger than the original orthogonal group $\mathrm{O}(2)$, see (2.4). Thus some care must be taken in the interpretation of the results. The additional (and spurious) symmetry is not broken by higher order terms in (4.3) and (4.4) because the original spatially resonant terms corresponding to wavenumbers $N$ and $N+1$, viz. $\bar{A}^{N} B^{N}$ and $\bar{B}^{N-1} A^{N+1}$, become exponentially small as $N \rightarrow \infty$ and hence are absent from Eq. (2.1). The absence of these terms is important since if present they would split the mixed mode branch into two, characterized by $(N+1) \phi_{A}-N \phi_{B}=0, \pi$, where $\phi_{A, B}$ are the phases of $A$ and $B$. In addition, there is the possibility of a transition between these two branches via a tertiary branch of traveling waves created at either end through parity-breaking bifurcations (Crawford et al, 1990). None of this behavior will be present here.

Depending on the sign of $\beta+\gamma$ one of the two equations (4.13) and (4.14) possesses solutions that blow up in finite time. This is to be expected since one of the two branches that come together at the bicritical point is necessarily subcritical. Thus no global attractor can be expected. However, some interesting albeit local dynamics are still possible. To pursue these, we first consider the steady states. 


\subsection{Steady states and their linear stability}

For the sake of simplicity, we assume that

$$
\beta+\gamma>0
$$

The case $\beta+\gamma<0$ yields similar results, since Eqs. (4.13) and (4.14) are invariant under the operation

$$
A \leftrightarrow B, \quad \hat{\gamma} \rightarrow-\hat{\gamma}, \quad \beta \rightarrow-\beta, \quad \hat{\gamma} \rightarrow-\hat{\gamma} .
$$

The pure mode solutions of Eqs. (4.13) and (4.14) are given by

$$
\left|A_{s}\right|^{2}=\frac{\hat{v}-\hat{\mu}+\hat{\delta}^{2} /(2 \pi)}{\beta+\gamma}, \quad\left|B_{s}^{2}\right|=0, \quad \text { and } \quad\left|A_{s}\right|^{2}=0, \quad\left|B_{s}^{2}\right|=\frac{\hat{v}+\hat{\mu}-\hat{\delta}^{2} /(2 \pi)}{\beta+\gamma}
$$

and correspond to standing waves with adjacent wavenumbers, while the mixed modes are given by

$$
\left(\left|A_{s}\right|^{2},\left|B_{s}^{2}\right|\right)=\frac{\left(2 \pi \hat{\mu}-\hat{\delta}^{2},-2 \pi \hat{\mu}+\hat{\delta}^{2}\right)}{2 \pi(\beta-\gamma)}+\frac{(\hat{\gamma}, \hat{\imath})}{(3 \beta+\gamma)}
$$

and correspond to spatially modulated standing waves. In the following we exclude the codimensiontwo points $\beta-\gamma=0$ and $3 \beta+\gamma=0$, and take $\hat{\mu}$ as the bifurcation parameter. It follows that the two types of pure modes in (4.18) only exist if $\hat{\mu}<\hat{\mu}_{0}$ and $\hat{\mu}>\hat{\mu}_{0}^{-}$, respectively, where

$$
\hat{\mu}_{0}^{ \pm}=\hat{\delta}^{2} /(2 \pi) \pm \hat{\imath}
$$

are the bifurcation points from the flat state, and that they bifurcate in opposite directions. Mixed modes only exist if

$$
\hat{v} /(3 \beta+\gamma)>0
$$

and $\min \left\{\hat{\mu}_{1}^{-}, \hat{\mu}_{1}^{+}\right\}<\hat{\mu}<\max \left\{\hat{\mu}_{1}^{-}, \hat{\mu}_{1}^{+}\right\}$, where

$$
\hat{\mu}_{1}^{ \pm}=\hat{\delta}^{2} /(2 \pi) \pm(\beta-\gamma) \hat{\nu} /(3 \beta+\gamma)
$$

are the bifurcation points from the branches of pure modes. The linear stability properties of these steady states are easily determined. The pure modes exhibit only steady-state instabilities, and these correspond to the bifurcation points to the mixed mode state. The first pure mode in (4.19) bifurcates subcritically and hence is unstable with respect to perturbations of like form (i.e., standing wave perturbations with $m=0$ ), while the second pure mode is asymptotically stable if $\hat{v}>0$ and either (i) $\hat{v}>0,3 \beta+\gamma>0$ and $\hat{\mu}_{0}^{-}<\hat{\mu}<\hat{\mu}_{1}^{-}$, or (ii) $\hat{v}>0,3 \beta+\gamma<0$ and $\hat{\mu}>\hat{\mu}_{0}^{-}$, or (iii) $\hat{v}<0$, $3 \beta+\gamma<0$ and $\hat{\mu}>\hat{\mu}_{1}^{-}$, and unstable otherwise (see Fig. 2). The latter instabilities are nothing but an instability of a supercritical pure mode with respect to perturbations in the form of an adjacent mode. Such secondary bifurcations generate mixed modes and these can in turn be either stable or unstable. The linear stability of such mixed modes is found by replacing $A$ and $B$ in (4.13) and (4.14) by $A_{s}\left(1+X_{1} \mathrm{e}^{\lambda \tau}+\bar{X}_{2} \mathrm{e}^{\bar{\lambda} \tau}\right)$ and $B_{s}\left(1+Y_{1} \mathrm{e}^{i \tau}+\bar{Y}_{2} \mathrm{e}^{\bar{i} \tau}\right)$, respectively, and linearizing. The resulting 
(a)

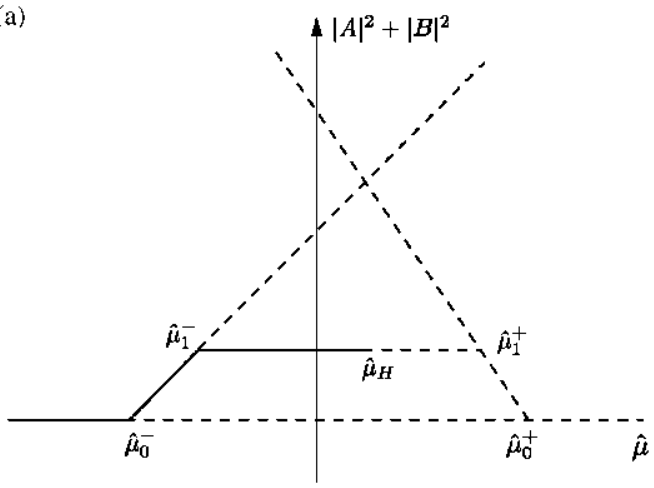

(c)

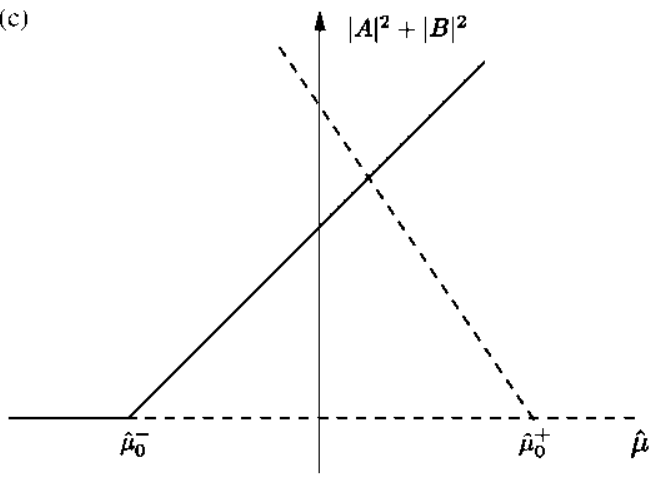

(b)

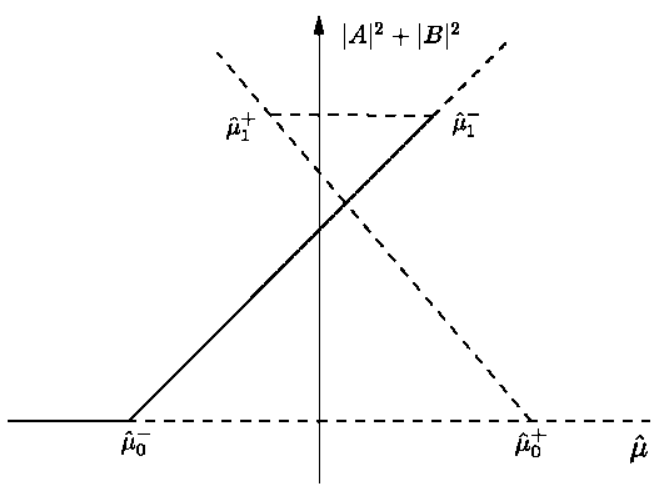

(d)

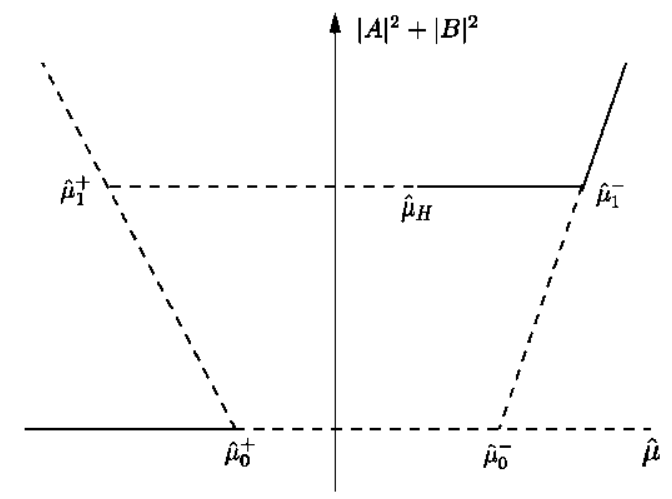

(e)

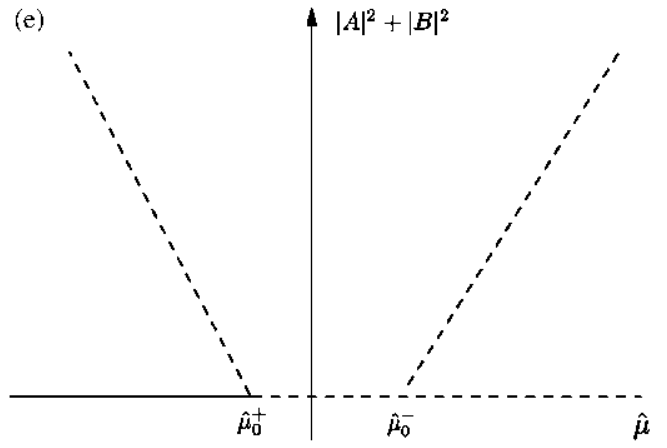

Fig. 2. Sketch of the stable (-) and unstable $(--)$ pure and mixed modes of $(4.13)$ for $\beta+\eta>0$ and: $(a) \hat{r}>0$, $3 \beta+\because>0, \beta-\because>0$, (b) $\hat{\imath}>0,3 \beta+\because>0, \beta-\because<0$, (c) $\hat{\imath}>0,3 \beta+\because<0$, (d) $\hat{\imath}<0,3 \beta+\because<0$ (thus $\beta-\because<0)$, and (e) $\hat{r}<0,3 \beta+\eta>0$. Results for $\beta+\eta<0$ follow using the symmetry (4.17).

algebraic equations

$$
\left[\frac{(\hat{i}+\hat{j})^{2}}{2 \pi}-\hat{\mu}+\hat{\imath}-2(\beta+\because)\left|A_{s}\right|^{2}-2 \beta\left|B_{s}\right|^{2}\right] X_{1}-(\beta+\because)\left|A_{s}\right|^{2} X_{2}-2 \beta\left|B_{s}\right|^{2}\left(Y_{1}+Y_{2}\right)=0,
$$




$$
\begin{aligned}
& -(\beta+\gamma)\left|A_{s}\right|^{2} X_{1}+\left[\frac{(\lambda+\hat{\delta})^{2}}{2 \pi}-\hat{\mu}+\hat{\gamma}-2(\beta+\gamma)\left|A_{s}\right|^{2}-2 \beta\left|B_{s}\right|^{2}\right] X_{2}-2 \beta\left|B_{s}\right|^{2}\left(Y_{1}+Y_{2}\right)=0, \\
& 2 \beta\left|A_{s}\right|^{2}\left(X_{1}+X_{2}\right)+\left[\frac{(\lambda+\hat{\delta})^{2}}{2 \pi}-\hat{\mu}-\hat{\gamma}+2(\beta+\gamma)\left|B_{s}\right|^{2}+2 \beta\left|A_{s}\right|^{2}\right] Y_{1}+(\beta+\gamma)\left|B_{s}\right|^{2} Y_{2}=0, \\
& 2 \beta\left|A_{s}\right|^{2}\left(X_{1}+X_{2}\right)+(\beta+\gamma)\left|B_{s}\right|^{2} Y_{1}+\left[\frac{(\lambda+\hat{\delta})^{2}}{2 \pi}-\hat{\mu}-\hat{\gamma}+2(\beta+\gamma)\left|B_{s}\right|^{2}+2 \beta\left|A_{s}\right|^{2}\right] Y_{2}=0,
\end{aligned}
$$

yield the characteristic equation

$$
\frac{\left(\hat{\lambda}^{2}+2 \hat{\delta} \lambda\right)^{2}}{4 \pi^{2}}\left[\left(\frac{\lambda^{2}+2 \hat{\delta} \lambda}{2 \pi}-2(\beta+\gamma)\left|A_{s}\right|^{2}\right)\left(\frac{\lambda^{2}+2 \hat{\delta} \lambda}{2 \pi}+2(\beta+\gamma)\left|B_{s}\right|^{2}\right)+16 \beta^{2}\left|A_{s}\right|^{2}\left|B_{s}\right|^{2}\right]=0,
$$

obtained with the help of (4.19). This equation possesses a double zero eigenvalue, with eigenfunctions

$$
\left(X_{1}, X_{2}, Y_{1}, Y_{2}\right)=\left(\mathrm{i} A_{s},-\mathrm{i} A_{s}, 0,0\right) \quad \text { and } \quad\left(X_{1}, X_{2}, Y_{1}, Y_{2}\right)=\left(0,0, \mathrm{i} A_{s},-\mathrm{i} A_{s}\right)
$$

which result from the invariance of $(4.13)$ and (4.14) under the actions (4.15). The remaining eigenvalues arising from the first factor are stable. Thus we only need to consider the roots of the second factor:

(i) If $\beta-\gamma \neq 0,3 \beta+\gamma \neq 0$ this factor yields zero eigenvalues only at the bifurcation points corresponding to the appearance of the branch of mixed modes.

(ii) However, purely imaginary eigenvalues, of the form $\lambda= \pm \mathrm{i} \hat{\lambda}_{I}$, are possible provided that

$$
\lambda_{I}^{2} / 2=\pi(\beta+\gamma)\left(\left|B_{s}\right|^{2}-\left|A_{s}\right|^{2}\right)=-\hat{\partial}^{2}+\left[\hat{\partial}^{4}+4 \pi^{2}(3 \beta+\gamma)(\beta-\gamma)\left|A_{s}\right|^{2}\left|B_{s}\right|^{2}\right]^{1 / 2}>0 .
$$

Since, according to $(4.19)$,

$$
\left|B_{s}\right|^{2}-\left|A_{s}\right|^{2}=-\frac{2\left[\hat{\mu}-\hat{\delta}^{2} /(2 \pi)\right]}{\beta-\gamma}, \quad\left|A_{s}\right|^{2}\left|B_{s}\right|^{2}=\frac{\hat{\gamma}^{2}}{(3 \beta+\gamma)^{2}}-\frac{\left[\hat{\mu}-\hat{\delta}^{2} /(2 \pi)\right]^{2}}{(\beta-\gamma)^{2}},
$$

we conclude immediately that the corresponding secondary Hopf bifurcation does not occur if $(3 \beta+\gamma)(\beta-\gamma)<0$. If however, $(3 \beta+\gamma)(\beta-\gamma)>0$ and $(4.21)$ holds, a unique Hopf bifurcation point $\hat{\mu}=\hat{\mu}_{H}$ is present. The uniqueness of this point follows from an examination of the two quantitics appcaring in Eq. (4.29) as a function of $M \equiv\left[\hat{\mu}-\hat{\delta}^{2} /(2 \pi)\right] /(\beta-\gamma)$ in the permitted range between $\pm \hat{v} /(3 \beta+\hat{\gamma})$.

The resulting bifurcation diagrams are sketched in Fig. 2, and are in agreement with general (but local) results for the (nonresonant) interaction of two period-doubling modes in the presence of $O(2)$ symmetry, cf. Crawford et al. (1990). Note, however, that for reasons already explained there 


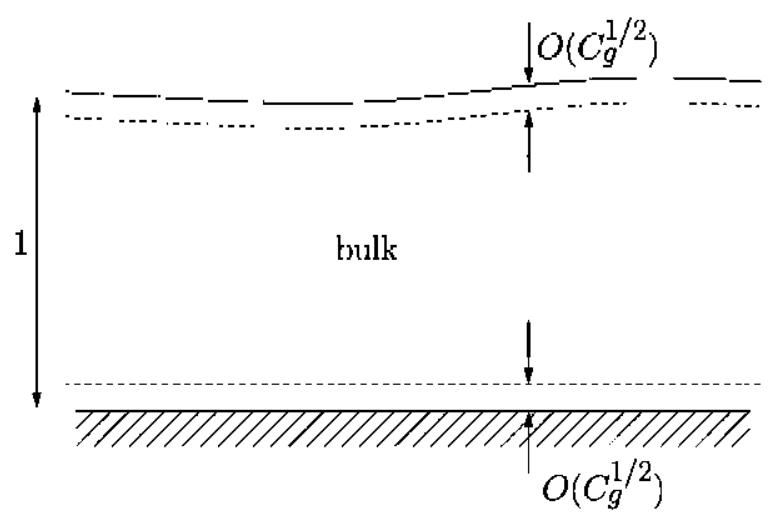

Fig. 3. Sketch of the lluid domain.

is only a single branch of mixed modes, and that this branch has two zero eigenvalues instead of the expected single zero eigenvalue. In particular, since there are no other eigenvalues in the phase direction, there are no parity-breaking bifurcations from the mixed mode branch. However, the Hopf bifurcation from the mixed modes does not require the presence of the resonant terms, since it occurs in the standing wave invariant subspace.

\section{Application to the Faraday system}

As mentioned in Section 1, Eqs. (2.1) and (2.2) describe Faraday waves in large aspect ratio, two-dimensional containers, provided that the effect of the mean flow produced by the waves is included. The proper form of this mean flow can be obtained from the general coupled amplitude mean flow (GCAMF) equations, derived in Vega et al. (2001). As shown below, the mean flow has only a minor effect in the generic case considered in Section 3, but its effect near the bicritical point considered in Section 4 is much more dramatic.

\subsection{Coupled amplitude-mean flow equations}

We consider a two-dimensional fluid layer above a horizontal plate that is vibrated vertically with an appropriately small amplitude (Fig. 3). The layer is laterally unbounded, with periodic boundary conditions. We use a Cartesian coordinate system with the $x$-axis along the unperturbed free surface and $y$ vertically upwards, and nondimensionalize space and time with the unperturbed depth $h$ and the gravity-capillary time $\left[g / h+T /\left(\rho h^{3}\right)\right]^{-1 / 2}$, where $g$ is the gravitational acceleration, $\rho$ is the density and $T$ is the coefficient of surface tension. The nondimensional equations governing the system then are (Vega ct al., 2001)

$$
\begin{aligned}
& \psi_{x x}+\psi_{y y}=\Omega, \quad \Omega_{t}-\psi_{y} \Omega_{x}+\psi_{x} \Omega_{y}=C_{y}\left(\Omega_{x x}+\Omega_{y y}\right), \\
& f_{l}-\psi / \psi_{x}-\psi_{y} f_{x}=\left(\psi_{y y}-\psi_{x x}\right)\left(1-f_{x}^{2}\right)-4 f_{x} k_{y y}=0 \quad \text { at } y=f,
\end{aligned}
$$




$$
\begin{aligned}
& (1-S) f_{x}-S\left(f_{x} / \sqrt{1+f_{x}^{2}}\right)_{x x}-\psi_{y t}+\psi_{x t} f_{x}-\left(\psi_{x}+\psi_{y} f_{x}\right) \Omega+\left(\psi_{x}^{2}+\psi_{y}^{2}\right)_{x} / 2 \\
& \left.+\left(\psi_{x}^{2}+\psi \psi_{y}^{2}\right)_{y} f_{x} / 2-4 \mu \alpha\right)^{2} \sin (2 \omega t) f_{x}=-C_{y}\left[3 / k_{x y}+\psi_{y y y}-\left(\psi_{x x}+\psi_{x y y}\right) f_{x}\right] \\
& +2 C_{y}\left[\frac{2 \psi_{x y} f_{x}^{2}+\left(\psi_{x x}-\psi_{y y}\right) f_{x}}{1+f_{x}^{2}}\right]_{x}+2 C_{y} \frac{\left(\psi_{x x y}-\psi_{y y y}\right) f_{x}^{2}-\psi_{x y y}\left(1-f_{x}^{2}\right) f_{x}}{1+f_{x}^{2}} \text { at } y=f \text {, } \\
& \int_{0}^{l} \Omega_{y} \mathrm{~d} x=\psi=\psi_{y}=0 \quad \text { at } y=-1 \\
& \psi(x+L, y, t) \equiv \psi(x, y, t), \quad f(x+L, t) \equiv f(x, t), \quad \int_{0}^{L} f \mathrm{~d} x=0 .
\end{aligned}
$$

Here $\psi$ is the streamfunction. such that the velocity $(u, v)=\left(-\psi \psi_{y}, \psi_{i}\right), \Omega$ is the vorticity, and $f$ is the free surface elevation required to satisfy volume conservation recalled in $(5.5 \mathrm{c})$. Condition (5.4a) is necessary in order that the pressure be periodic in $x$. The remaining equations and boundary conditions are standard. The resulting problem depends on the aspect ratio $L$, the nondimensional vibration amplitude $\mu$ and frequency $2 \omega$, the capillary-gravity number $C_{g}=v /\left[g h^{3}+T h / \rho\right]^{1 / 2}$, and the gravity-capillary balance parameter $S=T /\left(T+\rho g h^{2}\right)$, where $v$ is the kincmatic viscosity. Note that $C_{g}$ and $S$ are related to the usual Onhesorge number $C=v[\rho /(T h)]^{1 / 2}$ and the Bond number $B=\rho g h^{2} / T$ by $C_{g}=C /(1+B)^{1 / 2}$ and $S=1 /(1+B)$. Thus $0 \leqslant S \leqslant 1$, and the extreme values, $S=0$ and 1 , correspond to the purcly gravitational $(T=0)$ and the purely capillary $(g=0)$ limits, respectively.

In a laterally unbounded layer the driving frequency $2 a$ selects a wavenumber $k$ near $k_{0}$, given by the inviscid dispersion relation for surface gravity-capillary waves

$$
a=\left[\left(1-S+S k_{0}^{2}\right) k_{0} \tanh k_{0}\right]^{1 / 2} .
$$

The associated eigenfunction is proportional to $(\psi f)=\left(\Psi_{0}^{\prime}, 1\right)$, with

$$
\Psi_{0}=\alpha \sinh \left[k_{0}(y+1)\right] /\left(k_{0} \sinh k_{0}\right) .
$$

The GCAMF equations are derived under the following assumptions:

$$
\left(1+k_{0}\right)\left(C_{y} / \alpha\right)^{1 / 2} \ll 1, \quad L \gg 1, \quad|k| x|+| \psi_{y}|\ll 1, \quad| f \mid \ll 1,
$$

and the assumption that the spatial Fourier transforms of $\psi$ and $f$ both peak for all time around the wavenumbers $\pm m k_{0}$, with $m=0,1, \ldots$. These require, in particular, that

$$
C_{g} \ll 1 .
$$

In a laterally bounded container the wavenumber $k_{0}$ may not fit, and instead the vertical vibration of the container will select wavenumbers near the slightly shifted wavenumber $k$, defined by

$$
k=2 \pi N / L,
$$

where $N \geqslant 1$ is an integer such that

$$
-\pi<k_{0} L-2 \pi N \leqslant \pi .
$$


Thus

$$
\left|k-k_{0}\right| \sim L^{-1} \ll 1 .
$$

With the wavenumber $k$ the inviscid problem can be embedded in one with periodic boundary conditions. Thus in the following we use $k$ instead of $k_{0}$ as the basic wavenumber.

The above assumptions permit us to decompose the streamfunction (and vorticity) in the bulk and the free surface elevation into three parts, namely, (i) two parametrically excited counter-propagating watetrains with frequency $o$ and wavenumbers $\pm k$ associated with the surface gravity-capillary modes, modulated slowly in both space and time, (ii) a mean flow depending weakly on time but in principle strongly on the spatial variables $x$ and $y$, and (iii) the remaining part of the solution, which will be called nonresonant. As discussed in Vega et al. (2001) our assumptions guarantec that the mean flow variables exhibit well-defined averages in the fast variable $x$ (see $(5.24)$ below). Under these conditions the free-surface deflection and the streamfunction in the bulk can be written in the form

$$
\begin{aligned}
& f=\mathrm{e}^{\mathrm{i} / m t}\left(A^{+} \mathrm{e}^{\mathrm{i} k x}+A^{-} \mathrm{e}^{-\mathrm{i} k x}\right)+\mathrm{c} . \mathrm{c} .+H O T+f^{m}+N R T \\
& \psi=\Psi_{0} \mathrm{c}^{\mathrm{i} \omega t}\left(A^{+} \mathrm{c}^{\mathrm{i} k x}-A^{-} \mathrm{c}^{-\mathrm{i} k x}\right)+\mathrm{c} . \mathrm{c}+H O T+\psi^{m}+N R T
\end{aligned}
$$

Here the superseript $m$ denotes the mean flow variables, and NRT and HOT stand for nonesonam terms and higher order terms, respectively. The function $\Psi_{0}$, defined in $(5.7)$, is evaluated at the shifted wavenumber $k$. The complex amplitudes $A^{ \pm}$depend weakly on both $l$ and $x$, while $f^{m}, \psi^{m}$ and $\Omega^{m}$ depend weakly on $t$ but strongly on $x$ (and $y$ ),

$$
\left|A_{x}^{ \pm}\right|+\left|A_{t}^{ \pm}\right| \ll\left|A^{ \pm}\right| \ll 1, \quad\left|f_{t}^{m}\right| \ll\left|f^{m}\right| \ll 1, \quad\left|\psi^{m}\right| \ll\left|\psi^{m}\right| \ll 1,
$$

cf. (2.3).

The complex amplitudes $A^{ \pm}$and the mean flow variables $\psi^{m}$ and $f^{m}$ evolve on a timescale that is large compared to the basic period $2 \pi / \alpha$ according to evolution equations obtained from appropriate solvability conditions (Vega et al., 2001). To adapt the resulting GCAMF equations to the notation and scaling used in (2.1), we must redefine $t, A^{ \pm}, \psi^{m}$, and $\mu$ as

$$
\tilde{t}=v_{g} t, \quad \tilde{A}^{ \pm}=A^{ \pm} / \sqrt{v_{g}}, \quad \tilde{\psi}^{m}=\psi^{m} / v_{y}, \quad \tilde{\mu}=\mu \omega k \tanh k / v_{y}
$$

where $v_{f f} \equiv \sigma^{\prime}(k)$ is the group velocity of the surface waves. Dropping tildes, the amplitude equations become

$$
\begin{aligned}
A_{t}^{ \pm} \mp A_{x}^{ \pm}= & \mathrm{i} \alpha A_{x x}^{ \pm}-(\delta+\mathrm{i} v) A^{ \pm}+\mathrm{i}\left(\beta\left|A^{ \pm}\right|^{2}+\gamma\left|A^{\mp}\right|^{2}\right) A^{ \pm}+\mathrm{i} \mu \bar{A}^{\mp} \\
& \pm \mathrm{i} \Gamma_{1} \int_{-1}^{0} g(y)\left\langle\psi_{y}^{m}\right\rangle^{x} \mathrm{~d} y A^{ \pm}+\mathrm{i} \Gamma_{2}\left\langle\zeta^{m}\right\rangle^{x} A^{ \pm}
\end{aligned}
$$

while the equation for the mean flow in the bulk takes the form

$$
\psi_{x x}^{m}+\psi_{y y}^{m}=\Omega^{m}, \quad \Omega_{t}^{n}-\left[\psi_{y}^{m}+\left(|A \cdot|^{2}-\left|A^{-}\right|^{2}\right) g(y)\right] \Omega_{x}^{m}+\psi_{x}^{m} \Omega_{y}^{n}=\left(C_{g} / v_{y}\right)\left(\Omega_{x x}^{m}+\Omega_{y y}^{n}\right),
$$

in $-1<y<0$, with the boundary conditions

$$
\psi_{x}^{m}-f_{t}^{m}=\Gamma_{3}\left(\left|A^{-}\right|^{2}-\left|A^{\dagger}\right|^{2}\right)_{x}, \quad \psi_{y y}^{m}=\Gamma_{4}\left(\left|A^{-}\right|^{2}-\left|A^{-}\right|^{2}\right) \quad \text { at } y=0,
$$




$$
\begin{aligned}
& (1-S) f_{x}^{m}-S f_{x x x}^{m}-v_{y}^{2} \psi_{y t}^{m}+C_{g} v_{g}\left(\psi_{y y y}^{m}+3 \psi_{x x y}^{m}\right)=-\Gamma_{5} v_{g}\left(\left|A^{+}\right|^{2}+\left|A^{-}\right|^{2}\right)_{x} \quad \text { at } y=0, \\
& \int_{0}^{L} \Omega_{y}^{m} \mathrm{~d} x=\psi^{m}=0, \quad \psi_{y}^{m}=-\Gamma_{6}\left[\mathrm{i} A^{+} \bar{A}^{-} \mathrm{e}^{2 \mathrm{i} k x}+\mathrm{c} . \mathrm{c} .+\left|A^{-}\right|^{2}-\left|A^{+}\right|^{2}\right] \quad \text { at } y=-1, \\
& A^{ \pm}(x+L, l) \equiv A^{ \pm}(x, l), \quad \psi^{m}(x+L, y, l) \equiv \psi^{m}(x, y, l), \quad f^{m}(x+L, l) \equiv f^{m}(x, l), \\
& \int_{0}^{L} f^{m}(x, t) \mathrm{d} x=0 .
\end{aligned}
$$

The latter are derived from a careful matching between the nonlinear flow in the oscillatory boundary layers (whose thickness is $O\left(C_{g}^{1 / 2}\right)$ ) and the flow in the bulk (Vega et al., 2001). The horizontal mean value $\langle\cdot\rangle^{x}$ is defined as

$$
\langle G(x, y, t)\rangle^{x}=(2 \ell)^{-1} \int_{x-\ell}^{x+\ell} G(z, y, t) \mathrm{d} z, \quad \text { with } 1 \ll \ell \ll L,
$$

while $\delta>0$ and $v$ are given by

$$
\delta=\left(\alpha_{1} C_{g}^{1 / 2}+\alpha_{2} C_{g}\right) / v_{g}, \quad v=\left[2 \pi N / L-k_{0}-v_{g} x_{1} C_{g}^{1 / 2}\right] / v_{g},
$$

where

$$
\alpha_{1}=k(o / 2)^{1 / 2} / \sinh (2 k), \quad \alpha_{2}=\left[2+\left(1+\tanh ^{2} k\right) /\left(4 \sinh ^{2} k\right)\right] k^{2} .
$$

Finally, the dispersion coefficient $\alpha$ is given by

$$
x=-\omega^{\prime \prime}(k) /\left(2 v_{q}\right) .
$$

The remaining coefficients in $(5.17),(5.19)-(5.21), \beta, \gamma, \Gamma_{1}, \ldots, \Gamma_{6}$, and the function $g$ have also been computed, and are given by

$$
\begin{aligned}
& \beta=\frac{\omega k^{2}\left[(1-S)\left(9-\sigma^{2}\right)\left(1-\sigma^{2}\right)+S k^{2}\left(7-\sigma^{2}\right)\left(3-\sigma^{2}\right)\right]}{4 \sigma^{2}\left[(1-S) \sigma^{2}-S k^{2}\left(3-\sigma^{2}\right)\right]}+\frac{\left[8(1-S)+5 S k^{2}\right] \omega k^{2}}{4\left(1-S+S k^{2}\right)}, \\
& \gamma=-\frac{\omega k^{2}}{2}\left[\frac{\left(1-S+S k^{2}\right)\left(1+\sigma^{2}\right)^{2}}{\left(1-S+4 S k^{2}\right) \sigma^{2}}+\frac{\left.4(1-S)+7 S k^{2}\right]}{1-S+S k^{2}}\right], \\
& \Gamma_{1}=k \sigma /(2 \omega), \quad \Gamma_{2}=\omega k\left(1-\sigma^{2}\right)\left(2 \sigma v_{y}\right) . \\
& \Gamma_{3}=2 \omega / \sigma, \quad \Gamma_{4}=8 \omega k^{2} / \sigma, \quad \Gamma_{5}=\left(1-\sigma^{2}\right) \omega^{2} / \sigma^{2}, \quad \Gamma_{6}=3\left(1-\sigma^{2}\right) \omega k / \sigma^{2} . \\
& g(y)=2 \omega k \cosh [2 k(y+1)] / \sinh ^{2} k,
\end{aligned}
$$

where $\sigma=\tanh k$. Note that $\beta$ diverges at $(1-S) \sigma^{2}=S k^{2}\left(3-\sigma^{2}\right)$, i.e., when the strictly inviscid eigenfrequency $(5.6)$ satisfies $r(2 k)=2 \sigma(k)$. In the present paper we do not pursue this $2: 1$ resonance further; see Jones (1992) and Christodoulides and Dias (1994) for a strictly inviscid analysis, and 
MeGoldrick (1970) and Trulsen and Mei (1995, 1997) for nearly inviscid descriptions that ignore the mean flow.

\subsection{Dynamics near the bicritical point}

To examine the role of the mean flow near the bicritical point we first rescale the variables and parameters as in (2.10), namely

$$
(x, t)=L(\tilde{x}, \tilde{t}), \quad A^{ \pm}=L^{-1} \tilde{A}^{ \pm}, \quad(v, \mu)=L^{-1}(\tilde{v}, \tilde{\mu}),
$$

and

$$
\psi^{m}=L^{-2} \tilde{\psi}^{m}, \quad f^{m}=L^{-2} \tilde{f}^{m},
$$

where $\tilde{\psi}^{m} \equiv \tilde{\psi}^{m}(\tilde{x}, y, \tau), f^{m} \equiv f^{m}(\tilde{x}, \tau)$. Dropping the tildes and defining the bifurcation parameters $\hat{\mu}$ and $\hat{v}$, the damping rate parameter $\hat{\delta}$ and the slow time variable $\tau$ as in (4.1) and (4.2), namely

$$
\mu=\pi+L^{-1}\left(\hat{\mu}-2 \pi^{2} \alpha\right), \quad v=\pi+L^{-1}\left(\hat{v}-2 \pi^{2} \alpha\right), \quad \hat{\delta}=L^{-3 / 2} \hat{\delta} . \quad \tau=L^{-1 / 2} l,
$$

Eqs. (5.17)-(5.23) bccomo

$$
\begin{aligned}
& A_{t}^{ \pm} \mp A_{x}^{ \pm}+\mathrm{i} \pi\left(A^{ \pm}-\bar{A}^{\mp}\right)=-L^{-1 / 2}\left(A_{\tau}^{ \pm}+\hat{\delta} A^{ \pm}\right)+\mathrm{i} L^{-1}\left[\left(\hat{\mu}-2 \pi^{2} \alpha\right) \bar{A}^{\mp}-\left(\hat{y}-2 \pi^{2} \alpha\right) A^{ \pm}\right] \\
& +\mathrm{i} L^{-1}\left[\left(\beta\left|A^{ \pm}\right|^{2}+\gamma\left|A^{\mp}\right|^{2}\right) A^{ \pm}+\alpha A_{x x}^{ \pm}\right] \\
& +L^{-1}\left[ \pm \mathrm{i} \Gamma_{1} \int_{-1}^{0} g(y)\left\langle\psi \psi_{y}^{m}\right\rangle^{x} \mathrm{~d} y+\mathrm{i} \Gamma_{2}\left\langle f^{m}\right\rangle^{x}\right] A^{ \pm}+\cdots, \\
& \psi_{y y \tau}^{m}=c \psi_{y y y y}^{m} \quad \text { in }-1<y<0 \\
& \psi_{x}^{m}=\Gamma_{3}\left(\left|A^{-}\right|^{2}-\left|A^{\prime}\right|^{2}\right)_{x}, \quad \psi_{y y}^{m}=\Gamma_{4}\left(\left|A^{\mid}\right|^{2}-\left|A^{-}\right|^{2}\right) \quad \text { at } y=0, \\
& \psi^{m}=0, \quad \psi_{y}^{m}=-I_{6}^{\prime}\left[\mathrm{i} A^{\mid} \bar{A}^{-} \mathrm{e}^{2 \mathrm{i} k L x}+\text { c.c. }+\left|A^{-}\right|^{2}-\left|A^{\mid}\right|^{2}\right] \quad \text { at } y=-1, \\
& A^{ \pm}(x+1, t, \tau) \equiv A^{ \pm}(x, t, \tau), \quad \psi^{m}(x+1, y, \tau) \equiv \psi^{m}(x, y, \tau),
\end{aligned}
$$

cf. (4.3) and (4.4), where, because of the scaling (5.33), the spatial average (5.24) must be replaced by

$$
\langle G(x, y, t)\rangle^{x}=(2 \ell)^{-1} \int_{x-\ell}^{x+\ell} G(z, y, t) \mathrm{d} z, \quad \text { with } 1 / L \ll \ell \ll 1 .
$$

The parameter $\varepsilon$ appearing in (5.37) is defined as

$$
\varepsilon=L^{3 / 2} C_{g /} / v_{q}
$$

and is always (at least logarithmically) small; see remark at the end of this section. Note that the free surface elevation $f^{m}(x, \tau)$ decouples from the remaining mean llow variables, and is determined 
by $(5.20)$ and $(5.23)$,

$$
f^{m}=-I_{5} v_{g}\left[\left|A^{+}\right|^{2}+\left|A^{-}\right|^{2}-\int_{0}^{1}\left(\left|A^{-}\right|^{2}+\left|A^{-}\right|^{2}\right) \mathrm{d} x\right] /(1-S),
$$

where the integral comes from volume conservation and we assumed

$$
1-S \sim 1
$$

thereby excluding the capillary limit.

As in Section 4, we now write

$$
A^{ \pm}=A_{0}^{ \pm}+L^{-1 / 2} A_{1}^{ \pm}+L^{-1} A_{2}^{ \pm}+\cdots+\left(B_{0}^{ \pm}+L^{-1 / 2} B_{1}^{ \pm}+L^{-1} B_{2}^{ \pm}+\cdots\right) \mathrm{e}^{ \pm 2 \mathrm{i} \pi x}+H O H
$$

where

$$
A_{0}^{-}=\bar{A}_{0}^{+}, \quad B_{0}^{-}=-\bar{B}_{0}^{+} .
$$

Thus

$$
\left|A^{+}\right|^{2}-\left|A^{-}\right|^{2}=2 \bar{A}_{0}^{+} B_{0}^{+} \mathrm{e}^{2 \mathrm{i} \pi x}+\text { c.c. }+\cdots, \quad\left|A^{+}\right|^{2}+\left|A^{-}\right|^{2}=2\left(\left|A_{0}^{+}\right|^{2}+\left|B_{0}^{+}\right|^{2}\right)+\cdots .
$$

and so $f^{m}$ vanishes at leading order. Likewise, we suppose that

$$
\psi^{m}=\psi_{0}(y, \tau) \mathrm{e}^{2 \mathrm{i} \pi x}+\psi_{1}(y, \tau) \mathrm{e}^{2 \mathrm{i} k / x}+\text { c.c. }+\cdots,
$$

although $\psi / 1$ will not be needed in what follows. The slow function $\psi / 0$ is found (upon substitution of (5.43), (5.47a) and (5.48) into (5.37)-(5.39)) to be given by

$$
\begin{aligned}
& \psi_{0 y y \tau}=c \psi_{0 y y y y} \quad \text { in }-1<y<0, \\
& \psi_{0}=-2 I_{3} \bar{A}_{0}^{\prime} B_{0}^{\prime}, \quad \psi_{0 y y}=2 I_{4} \bar{A}_{00}^{\prime} B_{0}^{\prime} \quad \text { at } y=0, \\
& \psi_{0}=0, \quad \psi_{0 y}=2 I_{6} \bar{A}_{0}^{\prime} B_{0} \quad \text { at } y=-1 .
\end{aligned}
$$

Eqs. (4.9) and (4.10) continue to hold, and again yield no solvability condition. However, Eqs. (4.11) and (4.12) must be replaced by

$$
\begin{aligned}
\mathrm{i} \pi\left(A_{2}^{ \pm}-\bar{A}_{2}^{\mp}\right)= & -\mathrm{d} A_{1}^{ \pm} / \mathrm{d} \tau-\hat{\delta} A_{1}^{ \pm}+\mathrm{i}\left(\hat{\mu}-2 \pi^{2} \alpha\right) \bar{A}_{0}^{\mp}-\mathrm{i}\left(\hat{v}-2 \pi^{2} \alpha\right) A_{0}^{ \pm}+\mathrm{i} \beta\left(\left|A_{0}^{ \pm}\right|^{2}+2\left|B_{0}^{ \pm}\right|^{2}\right) A_{0}^{ \pm} \\
& +\mathrm{i} \gamma\left[\left(\left|A_{0}^{\mp}\right|^{2}+\left|B_{0}^{\mp}\right|^{2}\right) A_{0}^{ \pm}+\bar{A}_{0}^{\mp} B_{0}^{\mp} B_{0}^{ \pm}\right] \pm \mathrm{i} \Gamma_{1} \int_{-1}^{0} g(y) \psi_{0 y}^{\mp} \mathrm{d} y B_{0}^{ \pm}, \\
\mathrm{i} \pi\left(-B_{2}^{ \pm}-\bar{B}_{2}^{\mp}\right)= & -\mathrm{d} B_{1}^{ \pm} / \mathrm{d} \tau-\hat{\delta} B_{1}^{ \pm}+\mathrm{i}\left(\hat{\mu}-2 \pi^{2} \alpha\right) \bar{B}_{0}^{\mp}-\mathrm{i}\left(\hat{v}+2 \pi^{2} \alpha\right) B_{0}^{ \pm}+\mathrm{i} \beta\left(\left|B_{0}^{ \pm}\right|^{2}+2\left|A_{0}^{ \pm}\right|^{2}\right) B_{0}^{ \pm} \\
& +\mathrm{i} \gamma\left[\left(\left|B_{0}^{\mp}\right|^{2}+\left|A_{0}^{\mp}\right|^{2}\right) B_{0}^{ \pm}+\bar{B}_{0}^{\mp} A_{0}^{\mp} A_{0}^{ \pm}\right] \pm \mathrm{i} \Gamma_{1} \int_{-1}^{0} g(y) \psi_{0 y}^{ \pm} \mathrm{d} y A_{0}^{ \pm},
\end{aligned}
$$

where $\psi_{0}^{ \pm}$are defined as

$$
\psi_{0}^{\prime}=\psi_{0} \text { and } \psi_{0}^{-}=\bar{\psi}_{0} \text {. }
$$


Eqs. (5.52) and (5.53) are solvable only if the following conditions hold (ef. (4.13) and (4.14)):

$$
\begin{aligned}
& A^{\prime \prime}+2 \hat{\delta} A^{\prime}+\hat{\delta}^{2} A=2 \pi\left[\hat{\mu}-\hat{v}+\beta\left(|A|^{2}+2|B|^{2}\right)+\hat{\gamma}|A|^{2}\right] A+2 \pi \Gamma_{1} \int_{-1}^{0} g(y) \bar{\varphi}_{y} \mathrm{~d} y B \\
& B^{\prime \prime}+2 \hat{\delta} B^{\prime}+\hat{\delta}^{2} B=2 \pi\left[\hat{\mu}+\hat{v}-\beta\left(|B|^{2}+2|A|^{2}\right)-y|B|^{2}\right] B-2 \pi I^{\prime} \int_{-1}^{0} g(y) \varphi_{y} \mathrm{~d} y A
\end{aligned}
$$

where, as in Section 4 ,

$$
A=A_{0}^{\prime}=\bar{A}_{0}^{-}, \quad B=B_{0}^{\dagger}=-\bar{B}_{0}^{-}, \quad \varphi=\psi / \psi_{0} .
$$

Using these variables the mean flow equations (5.49)-(5.51) become

$$
\begin{aligned}
& \varphi_{y y \tau}=\delta \varphi_{y y y y} \quad \text { in }-1<y<0, \\
& \varphi=-2 I_{3} \bar{A} B, \quad \varphi_{y y}=2 I_{4} \bar{A} B \quad \text { at } y=0, \\
& \varphi=0, \quad \varphi_{y}=2 \Gamma_{6} \bar{A} B \quad \text { at } y=-1 .
\end{aligned}
$$

In the following we refer to (5.55), (5.6), (5.58)-(5.60) as the coupled amplitude-mean flow (CAMF) equations, and note that

- The CAMF equations are still invariant under the operations

$$
A \rightarrow-A, \quad A \rightarrow \mathrm{e}^{\mathrm{i} c_{1}} A, \quad \varphi \rightarrow \mathrm{e}^{-\mathrm{i} c_{1}} \varphi \quad \text { and } \quad B \rightarrow-B, \quad B \rightarrow \mathrm{e}^{\mathrm{i} c_{2}} B, \quad \varphi \rightarrow \mathrm{e}^{\mathrm{i} c_{2}} \varphi
$$

for all $c_{1}$ and $c_{2}$. Thus the spurious symmetry, noted in Section 4 , is still present. Moreover, since the mean flow is not slaved to the surface waves it plays a dynamical role in the stability of such waves (see below), and hence in their dynamics.

- When $|\hat{\hat{j}}| \sim 1$, as assumed above, the parameter $\varepsilon=\hat{\hat{\partial}} C_{g} /\left(\hat{\partial} v_{g}\right)$, and hence (using (5.6) and (5.25a)) is (i) of the order of $C_{g}^{1 / 2} \ll 1$ if $k$ is bounded above, and (ii) of the order of $1 / k^{2} \ll 1$ if $k$ is large. In fact, $\varepsilon$ is only logarithmically small (and so can be treated as $O(1)$ ) if $k$ is logarithmically large compared to $1 / C_{g}$.

- The CAMF equations show readily that the (leading order) mean flow is nonzero whenever $\bar{A} B \neq$ 0 , i.e., the mean flow vanishes only for the pure modes with $m=0$ or 1 .

\subsection{Linear stability of the pure modes}

The pure modes generate no mean flow and hence are still given by (4.18), namely

$$
\begin{gathered}
\left|A_{s}\right|^{2}=\frac{\hat{v}-\hat{\mu}+\hat{\delta}^{2} /(2 \pi)}{\beta+\gamma}, \quad\left|B_{s}^{2}\right|=\varphi_{s}=0, \\
\left|A_{s}\right|^{2}=\varphi_{s}=0, \quad\left|B_{s}\right|^{2}=\frac{\hat{v}+\hat{\mu}-\hat{\delta}^{2} /(2 \pi)}{\beta+\gamma} .
\end{gathered}
$$

In the context of the Faraday system these solutions represent pure standing waves, with respectively, $N$ and $N+1$ wavelengths in the period $L$. Fig, 4 a shows the regions in the $(S, k)$ plane where the quantity $\beta+\gamma$ is positive (unshaded) and negative (shaded). As in Section 4, wo assume that

$$
\beta+\gamma>0
$$



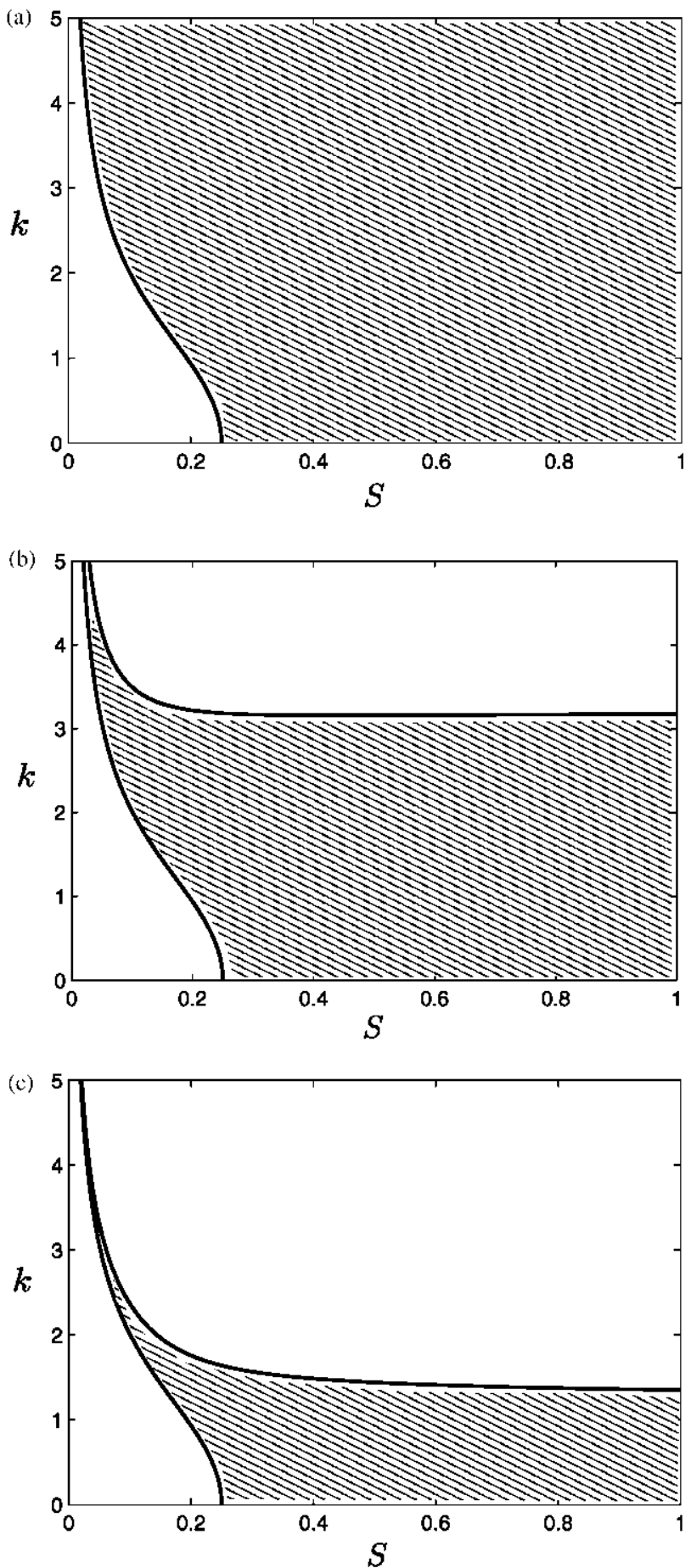

Fig. 4. The regions of positive (unshaded) and negative (shaded) values of the quantities (a) $\beta+\eta,(b) 3 \beta+\eta+\mathscr{c}(0)$ and (c) $\beta-;+f(0)$ in the $(S, k)$ plane. The curves bounding the shaded regions on the left are all distinet. 
The results for the case $\beta+\gamma<0$ follow from those below using the invariance of the CAMF equations under the operation

$$
A \leftrightarrow B, \quad \hat{v} \rightarrow-\hat{v}, \quad \beta \rightarrow-\beta, \quad \gamma \rightarrow-\hat{\gamma}, \quad \Gamma_{1} \rightarrow-\Gamma_{1} \quad \varphi \rightarrow \bar{\varphi} .
$$

The first of the pure modes in (5.62) then bifurcates subcritically and hence is always unstable. The linear stability properties of the second pure mode in (5.62) are determined by replacing $A$ by $B_{s}\left(X_{1} \mathrm{e}^{\lambda \tau}+\bar{X}_{2} \mathrm{e}^{\bar{\lambda} \tau}\right), B$ by $B_{s}\left(1+Y_{1} \mathrm{e}^{\lambda \tau}+\bar{Y}_{2} \mathrm{e}^{\bar{\lambda} \tau}\right)$ and $\varphi$ by $2\left|B_{s}\right|^{2}\left(\Phi X_{2} \mathrm{e}^{\lambda \tau}+\bar{\Phi} \bar{X}_{1} \mathrm{e}^{\bar{\lambda} \tau}\right)$ and linearizing Eqs. (5.55), (5.56) and (5.58)-(5.60). There are then two types of potential instabilities. The pure mode instability satisfics

$$
\begin{aligned}
& {\left[\frac{(\lambda+\hat{\delta})^{2}}{2 \pi}-\hat{\mu}-\hat{v}+2(\beta+\gamma)\left|B_{s}\right|^{2}\right] Y_{1}+(\beta+\gamma)\left|B_{s}\right|^{2} Y_{2}=0,} \\
& (\beta+\gamma)\left|B_{s}\right|^{2} Y_{1}+\left[\frac{(\lambda+\hat{\delta})^{2}}{2 \pi}-\hat{\mu}-\hat{v}+2(\beta+\gamma)\left|B_{s}\right|^{2}\right] Y_{2}=0,
\end{aligned}
$$

with $X_{1}, X_{2}$ and $\varphi$ slaved to $Y_{1}$ and $Y_{2}$. This system possesses a zero eigenvalue, with eigenfunction $\left(Y_{1}, Y_{2}\right)=\left(\mathrm{i} B_{s},-\mathrm{i} B_{s}\right)$, due to the invariance of Eqs. (5.55)-(5.60) with respect to the operation (5.61b). The remaining three eigenvalues are strictly negative, as in Section 4 . The mixed mode instability excites perturbations that are orthogonal to the mode in question. Thus $X_{1} \neq 0, X_{2} \neq 0$, but $Y_{1}=Y_{2}=0$. Both $X_{1}$ and $X_{2}$ satisfy identical equations:

$$
\left[\frac{(\lambda+\hat{\delta})^{2}}{2 \pi}-\hat{\mu}+\hat{v}-2 \beta\left|B_{s}\right|^{2}-2 \Gamma_{1}\left|B_{s}\right|^{2} \int_{-1}^{0} g(y) \Phi_{y} \mathrm{~d} y\right] X=0
$$

where $\Phi$ is the unique solution of

$$
\begin{aligned}
& \hat{\lambda} \Phi_{y y}=\varepsilon \Phi_{y y y y} \quad \text { in }-1<y<0, \\
& \Phi=-\Gamma_{3}, \quad \Phi_{y y}=\Gamma_{4} \quad \text { at } y=0, \\
& \Phi=0, \quad \Phi_{y}=I_{6} \quad \text { at } y=-1 .
\end{aligned}
$$

Integration of $(5.68)-(5.70)$ for $\lambda>0$ yields

$$
\Phi=K_{1}\left[\sinh \frac{\sqrt{\lambda}(y+1)}{\sqrt{\varepsilon}}-\frac{\sqrt{\lambda}(y+1)}{\sqrt{\varepsilon}}\right]-K_{2}\left[\cosh \frac{\sqrt{\lambda}(y+1)}{\sqrt{\varepsilon}}-1\right]+\Gamma_{6}(y+1),
$$

where

$$
\begin{aligned}
& K_{1}=\frac{\left(I_{3}+I_{6}\right) \cosh \sqrt{\lambda / \varepsilon}+I_{4}(\varepsilon / \lambda)[\cosh \sqrt{\lambda / \varepsilon}-1]}{\sqrt{\lambda / \varepsilon} \cosh \sqrt{\lambda / \varepsilon}-\sinh \sqrt{\lambda / c}}, \\
& K_{2}=\frac{\left(I_{3}+I_{6}\right) \sinh \sqrt{\lambda / 8}+I_{4}(\theta / \lambda)[\sinh \sqrt{\lambda / r}-\sqrt{\lambda / 6}]}{\sqrt{\lambda / \varepsilon} \cosh \sqrt{\lambda / \varepsilon}-\sinh \sqrt{\lambda / \varepsilon}},
\end{aligned}
$$

while if $\lambda=0$

$$
\Phi=\Gamma_{6}(y+1)-\frac{1}{4}\left[6\left(\Gamma_{3}+\Gamma_{6}\right)+\Gamma_{4}\right](y+1)^{2}+\frac{1}{4}\left[2\left(\Gamma_{3}+\Gamma_{6}\right)+\Gamma_{4}\right](y+1)^{3} .
$$


Note that all the eigenvalues $\lambda$ of (5.67) are doubled. These eigenvalues satisfy

$$
\frac{\lambda^{2}+2 \hat{\delta} \lambda}{2 \pi}+2 \hat{v}-(3 \beta+\gamma)\left|B_{s}\right|^{2}-\mathscr{R}(\lambda)\left|B_{s}\right|^{2}=0,
$$

where

$$
\mathscr{D}(\lambda) \equiv 2 \Gamma_{1} \int_{-1}^{0} g(y) \Phi_{y} \mathrm{~d} y
$$

Thus, if $\lambda>0$,

$$
\mathscr{D}(\lambda)=\frac{4 k^{2}}{\sinh 2 k}\left[\left(\Gamma_{6} \mathscr{W}_{1}+K_{1} \mathscr{W}_{2}(\lambda)-K_{2} \mathscr{W}_{3}(\lambda)\right],\right.
$$

but when $\lambda=0$,

$$
\mathscr{D}(0)=\frac{4 k^{2}}{\sinh 2 k}\left[I_{6} \mathscr{O}_{1}+\frac{1}{2}\left[6\left(I_{3}+I_{6}\right)+I_{4}\right] \mathscr{D}_{4}+\frac{3}{4}\left[2\left(I_{3}+I_{6}\right)+I_{4}\right] \mathscr{D}_{5}\right] .
$$

Here

$$
\begin{aligned}
& \mathscr{D}_{1}=\frac{\sinh 2 k}{2 k}, \quad \mathscr{D}_{2}=\sqrt{\frac{\lambda}{\varepsilon}}\left[\frac{\sinh (2 k+\sqrt{\lambda / \varepsilon})}{2(2 k+\sqrt{\lambda / \varepsilon})}+\frac{\sinh (2 k-\sqrt{\lambda / \varepsilon})}{2(2 k-\sqrt{\lambda / \varepsilon})}-\mathscr{D}_{1}\right], \\
& \mathscr{D}_{3}=\sqrt{\frac{\lambda}{\varepsilon}}\left[\frac{\cosh (2 k+\sqrt{\lambda / \varepsilon})-1}{2(2 k+\sqrt{\lambda / \varepsilon})}-\frac{\cosh (2 k-\sqrt{\lambda / \varepsilon})-1}{2(2 k-\sqrt{\lambda / \varepsilon})}\right], \\
& \mathscr{D}_{4}=\frac{\cosh 2 k-1}{4 k^{2}}-\mathscr{D}_{1}, \quad \mathscr{W}_{5}=\frac{\sinh 2 k}{4 k^{3}}-\frac{\cosh 2 k}{2 k^{2}}+\mathscr{D}_{1} .
\end{aligned}
$$

One can check that, properly interpreted, the above expressions apply even when $\lambda<0$ or when it is complex. Note that $\beta, \Gamma_{3}, \Gamma_{4}$ and $\Gamma_{6}$ depend only on $k$ and $S$, while $\hat{\delta}$ and $\varepsilon$ are determined by the chosen values of $C_{q}$ and $L ; \hat{\mu}$ and $\hat{v}$ are of course the two parameters that unfold the bieritical point.

The characteristic equation (5.74) admits a stationary instability at

$$
\hat{v}=[3 \beta+\gamma+\mathscr{D}(0)]\left|B_{s}\right|^{2} / 2,
$$

and the instability sets in as $\left|B_{s}\right|$ increases if $3 \beta+\gamma+\mathscr{D}(0)>0$ and as $\left|B_{s}\right|$ decreases if $3 \beta+\gamma+$ $\mathscr{D}(0)<0$. The regions of positive (unshaded) and negative (shaded) values of $3 \beta+\gamma+\mathscr{D}(0)$ in the $(S, k)$ plane are shown in Fig. 4b. The instability is oscillatory with $\lambda=\mathrm{i} \lambda_{I}, \lambda_{I}>0$, if

$$
\left|B_{s}\right|^{2}=\frac{\hat{\delta} \lambda_{I}}{\pi \mathscr{D}_{I}\left(\lambda_{1}\right)}, \quad \hat{v}=\frac{\lambda_{I}^{2}}{4 \pi}+\frac{\hat{\delta} \lambda_{I}\left[3 \beta+\gamma+\mathscr{V}_{R}\left(\lambda_{I}\right)\right]}{2 \pi \mathscr{Q}_{1}\left(\lambda_{I}\right)},
$$

where we have written $\mathscr{D}\left(\lambda=\mathrm{i} \lambda_{1}\right) \equiv \mathscr{Q}_{R}\left(\lambda_{1}\right)+\mathrm{i} \mathscr{Q}_{I}\left(\lambda_{1}\right)$. Thus (i) when $3 \beta+\gamma>0$ and $\mathscr{D}(0)>0(\mathscr{D}(0)$ $<0$ ) the mean flow reduces (increases) the amplitude at which the steady-state instability sets in and vice versa, and (ii) the mean flow permits the presence of an oscillatory instability on the supereritical pure mode branch provided $\mathscr{Q}_{I}\left(\lambda_{I}\right)>0$. This bifurcation generates a mean flow that oscillates back and forth, and henee cannot oceur if such a flow is excluded from the formulation (cf. Section 4). When $\mathscr{D}_{I}\left(\lambda_{I}\right)>0 \mathrm{Eq}$. (5.79) provides a parametric representation of a curve in the $\hat{v}$ vs. $\left|B_{s}\right|^{2}$ plane of the type shown in Fig. 5. In Fig. 5a-d we show for four choices of $S$ and $k$ the 

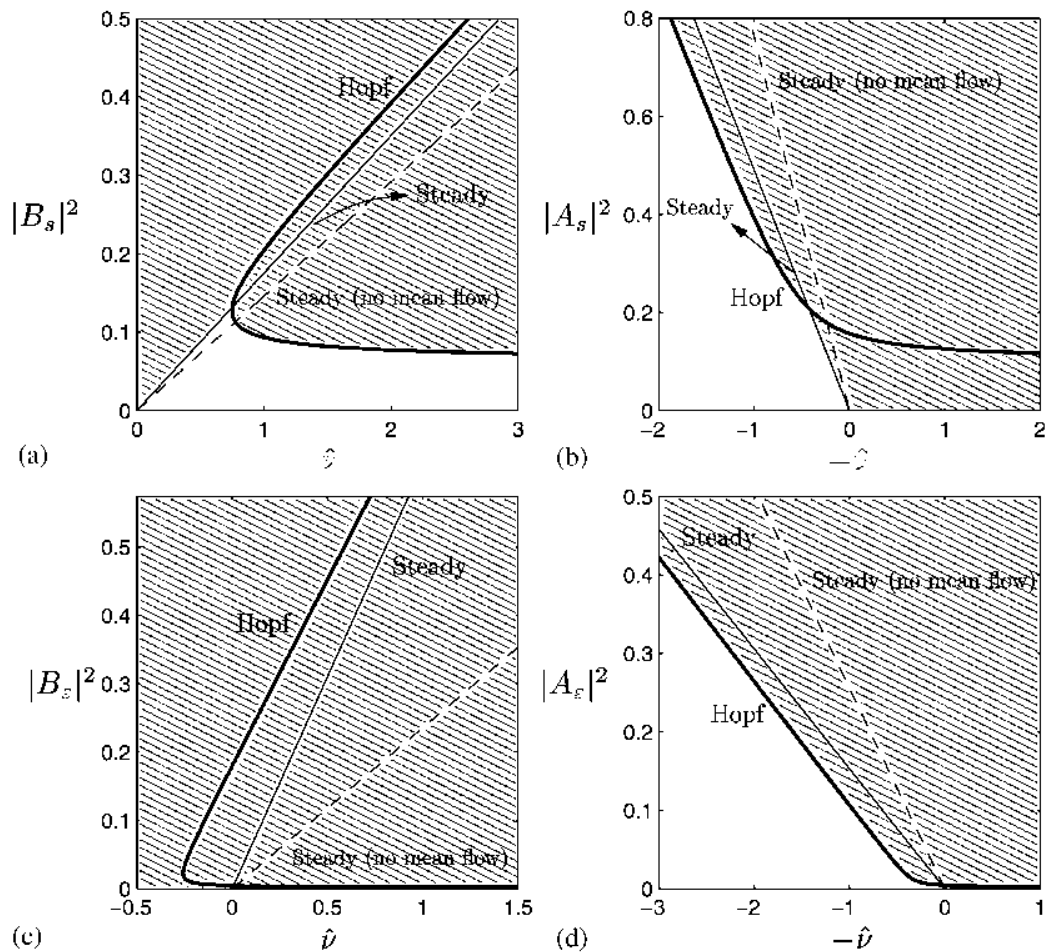

Fig. 5. The neutral steady and oscillatory instability curves of the second pure mode in (5.62), for $\hat{j}=1,:=0.1$ and (a) $k=0.5, S=0$. (b) $k=0.5, S=0.8$. (c) $k=2, S=0$. and (d) $k=1, S=0.8$. The pure mode is stable only below these curves. For comparison, the neutral instability curve without mean flow (i.e., $\mathscr{\ell}(0)=0)$ is shown using a dashed line. In all cases the results correspond to the bifurcation diagram in Fig. 2(a); to obtain this diagram for cases (b) and (d) one must first apply the transformation (5.64).

region in the $\left(\left|B_{s}\right|^{2}, \hat{v}\right)$ plane where the pure mode $\left(0 . B_{s}\right)$ is stable (unshaded) and unstable (shaded). Note that for sufficiently large detuning the presence of the Hopf bifurcation reduces dramatically the region of stability of the pure mode, especially in cases $(a, c)$. The reduction is smaller in cases (b,d); since $\beta+\because<0$ in these plots the transformation (5.64) has been applied.

\subsection{Linear stability of the mixed modes}

The mixed mode solutions of the CAMF equations (5.55), (5.56), and (5.58)-(5.60) are given by

$$
\left(\left|A_{s}\right|^{2},\left|B_{s}^{2}\right|\right)=\frac{\left(2 \pi \hat{\mu}-\hat{\delta}^{2},-2 \pi \hat{\mu}+\hat{\delta}^{2}\right)}{2 \pi[\beta-\imath+\mathscr{\imath}(0)]}+\frac{(\hat{\imath} \cdot \hat{\imath})}{3 \beta+\hat{\imath}+\mathscr{\lambda}(0)}, \quad\left(\rho_{s}=2 \Phi(0, \jmath) \bar{A}_{s} B_{s},\right.
$$

cf. Eq. (4.18a), where $\Phi(j ., 3)$ is the unique solution of $(5.68)-(5.70)$ and $\mathscr{\lambda}(0)$ is as defined in (5.77). In the following we exclude the codimension-two points $\beta-\eta+\mathscr{R}(0)=0$ and $3 \beta+\eta+\mathscr{Z}(0)=0$; both quantities are shown in Fig. $4 \mathrm{~b}$ and $\mathrm{c}$ in the $(S, k)$ plane. It follows that we may use Fig. 2 to deduce the structure (though not the stability properties) of the mixed mode branches, provided we replace $\beta-\eta$ and $3 \beta+\eta$ by $\beta-\eta+\mathscr{O}(0)$ and $3 \beta+\eta+\mathscr{O}(0)$, respectively. The steady-state 
bifurcation points at the end of the mixed mode branch are unchanged, although this is not true of the secondary Hopf bifurcation (see below).

The linear stability properties of the mixed modes are determined by replacing $A$, $B$, and $\varphi$ by $A_{s}\left(1+X_{1} \mathrm{e}^{\lambda \tau}+\bar{X}_{2} \mathrm{e}^{\bar{\lambda} \tau}\right), B_{s}\left(1+Y_{1} \mathrm{e}^{\lambda \tau}+\bar{Y}_{2} \mathrm{e}^{\bar{\lambda} \tau}\right)$, and $\varphi_{s}+\bar{A}_{s} B_{s}\left(\phi_{1} \mathrm{e}^{\lambda \tau}+\bar{\phi}_{2} \mathrm{e}^{\bar{\lambda} \tau}\right)$, respectively, and linearizing Eqs. $(5.55),(5.56)$ and $(5.58)-(5.60)$. From the latter it follows that:

$$
\phi_{1}=2\left(X_{2}+Y_{1}\right) \Phi(\lambda, y), \quad \phi_{2}=2\left(X_{1}+Y_{2}\right) \phi(\lambda, y),
$$

while the former show that $\left(X_{1}, X_{2}, Y_{1}, Y_{2}\right)$ satisfy the algebraic equation

$$
\begin{gathered}
{\left[\frac{(\lambda+\hat{\delta})^{2}}{2 \pi}-\hat{\mu}+\hat{\imath}-2(\beta+\gamma)\left|A_{s}\right|^{2}-2 \beta\left|B_{s}\right|^{2}\right] X_{1}-(\beta+\gamma)\left|A_{s}\right|^{2} X_{2}} \\
-[2 \beta+\grave{\lambda}(0)]\left|B_{s}\right|^{2} Y_{1}-2 \beta\left|B_{s}\right|^{2} Y_{2}-\mathscr{D}(\hat{\lambda})\left|B_{s}\right|^{2}\left(X_{1}+Y_{2}\right)=0,
\end{gathered}
$$

together with three equations obtained by (i) interchanging $X_{1} \leftrightarrow X_{2}$ and $Y_{1} \leftrightarrow Y_{2}$, (ii) interchanging $A_{s} \leftrightarrow B_{s}, X_{1} \leftrightarrow Y_{1}, X_{2} \leftrightarrow Y_{2}$, and changing the signs of $(\beta, \hat{\gamma}, \hat{v}, \mathscr{D})$, and (iii) interchanging $X_{1} \leftrightarrow X_{2}$ and $Y_{1} \leftrightarrow Y_{2}$ in the result of (ii). Here $(\lambda)$ is as defined in (5.76) and (5.77). Using (5.80) it follows that $X^{\prime} \equiv X_{1}+X_{2}$ and $Y^{\prime} \equiv Y_{1}+Y_{2}$ satisfy

$$
\begin{aligned}
& {\left[\frac{\lambda^{2}+2 \hat{\delta} \lambda}{2 \pi}-2(\beta+\gamma)\left|A_{s}\right|^{2}-(\mathscr{D}(\lambda)-\mathscr{D}(0))\left|B_{s}\right|^{2}\right] X^{-}-[4 \beta+\mathscr{D}(0)+\mathscr{D}(\hat{\lambda})]\left|B_{s}\right|^{2} Y^{\dagger}=0} \\
& {[4 \beta+\mathscr{D}(0)+\mathscr{D}(\lambda)]\left|A_{s}\right|^{2} X^{\dagger}+\left[\frac{\lambda^{2}+2 \hat{\delta} \lambda}{2 \pi}+2(\beta+\gamma)\left|B_{s}\right|^{2}+(\mathscr{Z}(\lambda)-\mathscr{D}(0))\left|A_{s}\right|^{2}\right] Y^{\dot{\lambda}}=0,}
\end{aligned}
$$

while $X^{-}=X_{1}-X_{2}$ and $Y^{-}=Y_{1}-Y_{2}$ satisfy

$$
\begin{aligned}
& {\left[\frac{\lambda^{2}+2 \hat{\delta} \lambda}{2 \pi}-(\mathscr{\lambda}(\lambda)-\mathscr{C}(0))\left|B_{s}\right|^{2}\right] X^{-}+[\mathscr{\lambda}(\lambda)-\mathscr{D}(0)]\left|B_{s}\right|^{2} Y^{-}=0,} \\
& -[\mathscr{\lambda}(\hat{\lambda})-\mathscr{D}(0)]\left|A_{s}\right|^{2} X^{-}+\left[\frac{\lambda^{2}+2 \hat{\delta} \lambda}{2 \pi}+(\mathscr{D}(\lambda)-\mathscr{D}(0))\left|A_{s}\right|^{2}\right] Y^{-}=0 .
\end{aligned}
$$

We may identify the former equations as describing instability with respect to amplitude or standing wave perturbations (the spatial phase of the mixed mode remains fixed by these perturbations), while the latter describe instability with respect to phase perturbations.

The nondegeneracy conditions $3 \beta+\gamma+\mathscr{L}(0) \neq 0, \beta-\gamma+\mathscr{L}(0) \neq 0$ (Fig. $4 \mathrm{~b}$ and $\mathrm{c}$ ) guarantee that the system (5.83) and (5.84) does not possess any zero eigenvalues; this is a consequence of the fact that the mixed mode branch is monotonic and henee contains no saddle-node bifurcations. Consequently, instability can only set in through a Hopf bifurcation. Since this bifurcation preserves spatial phase it produces a standing oscillation (i.e, a vacillation) about the mixed mode. In contrast, the system (5.85) and (5.86) always has a double zero eigenvalue (resulting from the symmetrics (5.61)), but in addition there can be a further zero eigenvalue resulting in a parity-breaking bifurcation of the mixed modes. To see this we examine the limit $\lambda \rightarrow 0$ of (5.85) and (5.86). In this limit 
$D(\lambda)-D(0)=\sigma \lambda+O\left(\lambda^{2}\right)$, and the system (5.85) and (5.86) reduces to a characteristic cquation of the form

$$
\lambda^{2}\left[\hat{\delta}-\frac{2 \pi \sigma\left(\hat{\mu}-\hat{\delta}^{2} / 2 \pi\right)}{\beta-\gamma+D(0)}+O(\lambda)\right]=0,
$$

indicating the presence of a parity-breaking bifurcation at

$$
\hat{\mu}=\hat{\mu}_{p} \equiv \frac{\hat{\delta}^{2}}{2 \pi}+\frac{\hat{\delta}}{2 \pi \sigma}[\beta-\gamma+D(0)] .
$$

This instability is only possible because the coupling to the mean flow results in a transcendental characteristic equation with a larger number of zero eigenvalues, and it produces traveling waves that drift steadily either to the left or the right. In addition we may have purely imaginary roots of the characteristic equation. This possibility is also a consequence of the coupling to the mean flow, and it generates the so-called direction-reversing waves, i.e., an oscillation in the spatial phase of the mixed mode (Landsberg and Knobloch, 1991). We do not pursue here these instabilities further.

\section{Concluding remarks}

In this paper we have examined the dynamics of the nearly inviscid Faraday system near codimension-two points where the neutral stability curves for adjacent modes cross. As is well-known such points provide the key to the nonlinear phenomena associated with the transition from one mode to another, since near such points the necessary secondary bifurcations all occur at small amplitude and so are (usually) analytically accessible. We focused on large aspect ratio domains which permit the presence of large-scale mean flow, and explored the role played by this flow in the resulting transition. In the absence of a mean flow we showed that the basic equations reduce to equations already familiar from the theory of nonresonant interaction of distinct modes. The different possibilities are summarized in Fig. 2. An important feature of these diagrams is that one of the two modes is necessarily subcritical while the other is supercritical. This property is a consequence of the fact that the primary Faraday resonance in an inviscid fluid produces an instability whose direction of branching depends on the sign of the detuning (relative to optimal).

In the absence of mean flow the only difference between the present theory and that appropriate to more viscous systems is the fact that the dynamics of the two interacting modes are second order in time. However, the added degrees of freedom are all damped and do not permit new types of instability. The situation changes dramatically once the coupling to the mean flow is included (Section 5). The mean llow shifts the onset of the secondary bifurcation to mixed modes (either increasing or decreasing the range of stable mixed modes, depending on parameters), but more importantly it also permits a new type of instability of the pure modes. This instability is oscillatory and produces the so-called direction reversing waves (Landsberg and Knobloch, 1991), i.e., periodic oscillation in the spatial phase of the pure mode. This instability may precede the steady-state instability to mixed mode, resulting in a dramatic change in the bifurcation behavior. Although we have not pursued the nonlinear evolution of these oscillations we surmise that they most likely disappear via a global bifurcation. Fig. 5 summarizes the possible secondary bifurcations from the pure mode branches as a function of the (scaled and shifted) detuning $\hat{v}$, with the regions of instability 
indicated by shading. In cases $(\mathrm{a}, \mathrm{c})$ the results of Fig. 4 show that $\beta+\gamma>0,3 \beta+\gamma+\mathscr{D}(0)>0$, $\beta-\gamma+\mathscr{D}(0)>0$, while in cases (b,d) $\beta+\gamma<0,3 \beta+\gamma+\mathscr{Z}(0)<0, \beta-\gamma+\mathscr{D}(0)<0$. The transformation (5.64) shows that the bifurcation diagrams in both cases are topologically the same provided one exchanges $B_{s}$ for $A_{s}$ and changes the sign of $\hat{v}$. This diagram is shown in Fig, 2a, and shows that the supercritical mode bifureates first and is initially stable. This mode loses stability at finite amplitude to a mixed mode which inherits the stability before undergoing a Hopf bifurcation. It is the first of these bifurcations that may be preceded by the bifurcation to direction-reversing waves. Fig. 5 also shows the presence of an interesting codimension-two point, at which the steady and Hopf bifurcations on the supercritical pure mode branch come in simultaneously. This is an interaction between a pitchfork and a symmetry-breaking Hopf bifurcation, and such interactions may lead to a variety of new dynamical phenomena, cf. Landsberg and Knobloch (1993).

Similar computations for the stability of the mixed modes in the presence of mean flow show that the mixed modes can lose stability in two ways, either via a parity-breaking steady-state bifurcation producing traveling waves, or via a Hopf bifurcation. Of the latter there are two types, a Hopf bifurcation in which the oscillations respect the spatial phase of the mixed modes (producing so-called standing waves) and a Hopf bifurcation which produces oscillations in the spatial phase (and so results in direction-reversing waves). Only the standing oscillations are possible in the absence of the mean flow (cf. Fig. 2a) and these must be present even with mean flow in diagrams such as Fig. $2 \mathrm{a}$ where (in the standing wave subspace) the stability assignments at the two ends of the mixed mode branch dilfer. In particular, in Fig. 5a the standing wave instability will necessarily destabilize the mixed modes in the region to the left of the codimension-two point. Although we have not pursued the mixed mode instabilities further it is clear that these permit the presence of new types of higher codimension points in parameter space (for example, where the two Hopf bifurcations coincide), and hence new types of rich dynamics.

\section{Acknowledgements}

This work was supported in part by the NASA Microgravity Sciences Program under Grant NAG3-2152 and by the National Science Foundation under Grant DMS-0072444. We are grateful to Dr. M. Higuera for providing Figs. 4 and 5.

\section{References}

Christodoulides, P., Dias, F, 1994. Resonant gravity-capillary interfacial waves. I. Fluid Mech, 265, 303-343.

Ciliberto, S., Gollub, J.P., 1985. Chaotic mode competition in parametrically forced surface waves. J. Fluid Mceh. 158, 381-398.

Craik, A.D.D., 1985. Wave Interactions and Fluid Flows. Cambridge University Press, Cambridge.

Crawford, J.D., Knobloch, li, Riecke, II, 1990. Period-doubling mode interactions with circular symmetry, Physica D 44, 340-396.

Cross, M.C., Hohenberg, P.C., 1993. Pattern formation outside of equilibrium. Rev. Mod. Phys. $65,8511112$.

Davey, A., Stewartson, S., 1974. On three-dimensional packets of surface waves. Proc. R. Soc. London A 338, 101-110.

Decent, S.P., Craik, A.D.D., 1999. Sideband instability and modulations of Faraday waves. Wave Motion $30,4355$.

Douady, S., Fauve, S., Thual, (o., 1989. Oscillatory phase modulation of parametrically forced surface waves. Iturophys. Lett. $10,309-315$. 
Faraday, M., 1831. On the forms and states assumed by fluids in contact with vibrating elastic surfaces. Phil. Trans. R. Soc. London 121,319340 .

Fauve, S., 1995. Parametric instabilities. In: Martínez-Mekler, (i., Seligman, T.H. (Eds.), Dynamics of Nonlinear and Disordered Systems. World Scientific, Singapore, pp. $67-115$.

Higuera, M., Vega, J,M., Knoblod, E, 2001. Interation ol" nearly-inviscid, multi-mode Faraday waves and mean flows. In: Bonilla, L.L., Platero, G., Regucra, D., Rubi, J.M. (Eds.), Coherent Structures in Complex Systems. Springer, Berlin, pp. 328337.

Ilignera, M., Nicolás, I.A., Vega. J.M., 2002a. Weakly nonlinear non-axisymmetric oscillations of capillary bridges at small viscosity. Plys. Fluids $14,3251-3271$.

TTignera, M., Porter, J., Knobloch, Г., 2002b. Teteroclinic dynamics in the nonlocal parametrically driven nonlinear Schrödinger equation. Physica D 162, 155-187.

Higuera, M., Vega, J.M., Knobloch, E., 2002c. Coupled amplitude-meen flow equations lor nearly-inviscid Faraday waves in moderate aspect ratio containers. J. Nonlinear Sci. 12, 505551.

Iskandarani, M., Lin, P.L.F., 1991. Mass transport in three-dimensional water waves. I. Filvid Mech, $231,417-437$.

Jones, M.C.W., 1992. Nonlinear stability of resonant capillary-gravity waves. Wave Motion 15, $267-283$.

Knobloch, E., Martel, C., Vega, J.M., 2002. Coupled mean llow-amplitude equations for nearly inviscid parametrically driven surface waves. Ann. N.Y. Acad. Sci. 974, 201219.

Knobloch, E., Vega, J.M., 2002. Nearly inviscid Faraday waves. In: Newton, P., Holmes, P., Weinstein, A. (Eds.), Geometry, Mechanics and Dymamics: Volume in Ilonor of the 60th Birthday of I.E., Marsden, Springer, 13erlin, pp. $181-222$.

Landsberg, A.S., Knobloch, E., 1991. Direction-reversing traveling waves. Phys. Letl. A 159, 17-20.

Landsberg, A.S., Knobloch, E., 1993. New types of waves in systems with O(2) symmetry. Phys. Lett. $\Lambda 179,316324$.

Lapuerta, V., Martel, C., Vega, J.M., 2002. Weakly dissipative Faraday waves in 2D large aspect ratio anmuli. Physica D 173, 178-203.

Liu, A.K., Davis, S.H, 1977. Viscous altenuation of mean drilt in water waves. J. Fluid Mech. 81, 63-84.

Longuet-Higgins, M.S., 1953. Mass transport in water waves. Phil. Trans. R. Soc. A 245, 535581.

Martel, C., Knobloch, E., Vega, J.M., 2000. Dynamics of counterpropagating waves in parametrically forced systems. Physica D $137,94-123$.

Martel, C., Vega, J.M., Knobloch, E., 2003. Dynamics of counlerpropagating waves in parametrically driven systems: dispersion vs. advection. Physica D 174, $198-217$

Martin, E., Martel, C., Vega. J.M., 2002. Drift instability of standing Faraday waves in an annular container. J. Fluid Mech, 467, 57-79.

Mocoldrick, L.F., 1970. On Wilton ripples: a special case of resonant interactions. I. Fluid Mech, 42, 193-200.

Miles, J., Henderson, D. 1990. Parametrically lored surlace waves. Annu. Rev. Fluid Mech. 22, $143-165$.

Pierce, R.D., Knobloch, E., 1994. On the modulational stability of traveling and standing water waves. Phys. Fluids 6, 11771190.

Rayleigh, Lord, 1883 . On the circulation of air observed in Kundt's tubes, and on some allied acoustical problems. Phil. Trans. R. Soc. London. 175, 1-21.

Rayleigh, Lord, 1945. The Theory of Sound, Vol. II. Dover, New York.

Riley, N., 2001. Steady streaming. Annu. Rev. Fluid Mech. 33, 4365.

Schlichting, H., 1932. Berechnung ebener periodischer Girenzschichtströmungen. Phys. Z. 33, 327335.

Schlichting, II, 1968. Bonndary Layer Theory. Mcciraw-llill, New York.

Simonelli, F, Gollub, J.P., 1989. Surlace wave mode interactions: effects of symmetry and degeneracy. J. Fluid Mech. 199, 471-494.

Trulsen, K., Mei, C.C., 1995. Modulation of three resonating gravity-eapillary waves by a long gravity wave. I. Fluid Mech. $290,345376$.

Trulsen, K., Mei, C.C., 1997. Effect of weak wind and damping on Wilton's ripples. J. Fluid Mech. $335,141163$.

Umeki, M., 1991. Haraday resonance in rectangular geometry. I. Fluid Mech. 227, 161-192.

Vege, J.M., Knobloch, E., Martel, C., 2001. Nearly inviscid Faraday waves in annular containers of moderately large aspect ratio. Physica D 154, 313-336. 